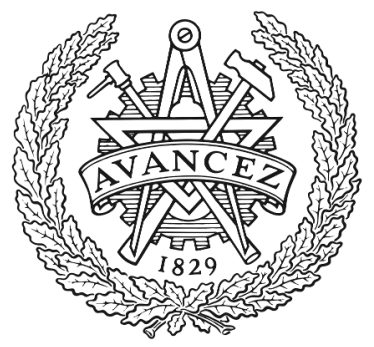

CHALMERS

UNIVERSITY OF TECHNOLOGY

\title{
Performance Analysis of Receivers using Sector Antennas for Broadcast Vehicular Communications
}

Downloaded from: https://research.chalmers.se, 2023-04-26 11:29 UTC

Citation for the original published paper (version of record):

Nagalapur, K., Brännström, F., Ström, E. (2019). Performance Analysis of Receivers using Sector Antennas for Broadcast Vehicular Communications. IEEE Transactions on Communications, 67(5): 3677-3692. http://dx.doi.org/10.1109/TCOMM.2019.2892040

N.B. When citing this work, cite the original published paper. 


\title{
Performance Analysis of Receivers using Sector Antennas for Broadcast Vehicular Communications
}

\author{
Keerthi Kumar Nagalapur, Fredrik Brännström, Member, IEEE, Erik G. Ström, Senior Member, IEEE
}

\begin{abstract}
In this paper, we analyze a carrier-sense multiple access (CSMA) system with all-to-all broadcast data traffic to assess the performance gain obtained by using multiple sector antennas and a receiver setup that can decode multiple packets simultaneously when packets arrive in narrow angle of arrivals. In the broadcast mode of IEEE 802.11p based vehicle-to-vehicle communications, acknowledgment messages are absent and a fixed contention window is used in medium access. As a result, the probability of multiple vehicles simultaneously transmitting a packet increases with the number of vehicles. In the case of a simultaneous transmission, a receiver with omnidirectional antennas receives power from all the transmitting vehicles and the probability of successfully decoding a packet decreases. This problem can be alleviated by using sector antennas when the simultaneously transmitted packets arrive at a receiver in narrow angle of arrivals. We show through analysis and simulations that the packet success rate (PSR) can be improved significantly by using the sector antennas setup instead of an omnidirectional antenna. Numerical results show that a several fold increase in PSR can be achieved in a setup with four sector antennas compared with an omnidirectional antenna when the density of vehicles is large.
\end{abstract}

Index Terms-Broadcast communications, sector antennas, dense networks, CSMA/CA, EDCA, packet success ratio

\section{INTRODUCTION}

Vehicle-to-vehicle (V2V) communications technology promises a multitude of vehicular applications without the requirement of a central coordinator. The absence of a central coordinator requires the vehicles to use a decentralized medium access control (MAC) mechanism to access the channel for transmitting a data packet. The MAC mechanism along with the physical (PHY) layer for vehicular communications has been standardized in ITS-G5 and DSRC in Europe and USA, respectively [1], [2]. Both standards have specified enhanced distributed channel access (EDCA) as the MAC scheme, which is an enhanced version of the carrier-sense multiple access with collision avoidance (CSMA/CA) scheme that imposes different priorities to different packet types [3, Section 9.19]. The V2V communications packets are broadly classified into periodic status messages and event triggered messages. Both kind of messages are all-to-all broadcast in nature, meaning that a transmitted packet is intended for all the receivers in

Keerthi Kumar Nagalapur, Fredrik Brännström, and Erik G. Ström are with the Communication Systems Group, Dept. of Electrical Engineering, Chalmers University of Technology, SE-412 96, Gothenburg, Sweden. E-mail: \{keerthi, fredrik.brannstrom,erik.strom\}@chalmers.se.

This research has been carried out in ChaseOn in a project financed by Swedish Governmental Agency of Innovation Systems (Vinnova), Chalmers, Bluetest, Ericsson, Keysight, RISE, Smarteq, and Volvo Cars. the transmission range. If all the receivers that decoded the packet were to report the success to the transmitter through a positive acknowledgment, the acknowledgments would collide at the transmitter. Therefore, positive acknowledgments of the CSMA/CA scheme are omitted in the broadcast mode. As a direct consequence of the absence of acknowledgments, CSMA/CA in the broadcast mode has only a single back-off stage, meaning that the contention window size is fixed and does not adapt to the number of packet collisions in the network. The fixed contention window sizes for each access class (AC) in the broadcast mode are specified in [4, Table B.6]. For the window sizes specified in the standard, it has been shown that the throughput of the system decreases significantly when the number of transceivers increases beyond a threshold [5].

Urban intersections and highways are two common scenarios where the number of vehicles in the transmission range can be large. The large number of vehicles in the intersection scenario is due to the high density of vehicles, while in the highway scenarios it is due to the larger coverage area which is a consequence of absence of buildings, few dominant scatterers, and high probability of line-of-sight (LOS) links [6], [7]. The high probability of LOS links and few dominant scatterers in the highway scenario implies that when two or more simultaneous transmissions occur they can arrive at the receiver with different angle of arrivals (AOAs) and narrow angular spreads. The directional arrival of packets can be exploited to reduce the number of collisions in the highway scenario. In this work, we aim to show the benefit of using sector antennas at the receiver in dense scenarios with high probability of LOS component, in particular, in highway scenarios. It should be emphasized, however, that the proposed scheme also works in non-LOS environments, as discussed in more detail in Section V.

Cellular-V2X device-to-device communications, outside the cellular network coverage, rely on transmitting resource reservation messages and booking resources for a time window (semi-persistent scheduling) to prevent simultaneous packet transmission [8], [9]. This work focuses on the EDCA medium access in $802.11 \mathrm{p}$ based vehicular communications where using sector antennas to prevent packet collisions is relatively more beneficial due to the absence of any resource reservation.

\section{A. Previous Work}

The idea of exploiting the directional arrival of packets in dense ad hoc networks has been considered before. Majority of the related works focus on reducing collisions in unicast 
communications by employing sector antennas and designing directional CSMA/CA schemes. The schemes propose to use different variations of directional request to send (RTS) and clear to send (CTS) messages to request channel access in specific sectors [10]-[14]. The proposed schemes have been shown to improve the throughput of ad hoc networks in comparison to omnidirectional transmission and reception. Since the V2V messages are all-to-all broadcast messages and no handshake messages are allowed, schemes involving directional RTS and CTS cannot be used. Furthermore, directional transmission of packets is not favorable as a packet from each transmitter is intended for all the other vehicles in the transmission range. However, it is possible to exploit sector antennas pointing in different directions on vehicles to reduce the number of packet collisions in comparison to an omnidirectional antenna and to also decode several packets arriving from different AOAs simultaneously. This does not require any modification to the MAC scheme specified in the standard. In [15], directional patch antennas are proposed for wireless sensor networks and it is shown that the communication range can be increased and the interference can be reduced by antenna selection. In [16], a roadside relay station located at the center of an urban intersection is equipped with sector antennas pointing towards each road. A collision model where two or more simultaneous packet transmissions are treated as packet collisions is used to show that more packets can be successfully decoded at the station when it is capable of decoding multiple packets.

The use of multiple antennas on vehicles to improve the diversity gain has been well explored [7], [17]. In [7], the benefit of using multiple antennas on vehicles for increasing diversity gain has been shown using a tool that uses ray tracing in combination with environment and traffic models. In [17], it has been shown through measurements that a pair of antennas with complementing properties improves the reception performance in vehicular environments. To utilize the full diversity gain offered by $J$ antennas in highly time-varying channels such as the vehicular channels, the receiver analog front end requires $J$ RF chains. The analog front end required at a receiver for decoding multiple packets simultaneously using $J$ antennas also requires $J$ RF chains. However, the digital signal processing stage following the analog to digital converters (ADCs) has to be designed to decode upto $J$ packets. Therefore, there is a possibility of using multiple sector antennas on vehicles for diversity gain in fading scenarios and to decode more than a single packet simultaneously in dense vehicular scenarios with high probability of LOS links. While the use of multiple antennas for diversity gain has been thoroughly investigated in the literature, a detailed performance analysis of using sector antennas to decode multiple packets simultaneously is missing, especially in the all-to-all broadcast scenario. In the all-to-all broadcast scenario, a nontransmitting vehicle, henceforth referred to as the receiver, is interested in all the messages being transmitted. As a consequence, it is beneficial for the receiver to decode a packet that is the most favorable from a signal to interference plus noise power ratio (SINR) perspective.

The main contributions of the paper are:
- We analyze the performance of sector antennas setup to decode multiple packets in the all-to-all broadcast scenario.

- The SINR is modeled as the ratio of the largest received power to the sum of the remaining received powers plus noise power. By fixing the minimum SINR threshold required to successfully decode a packet, we compute the average number of packets that are successfully decoded per packet duration, henceforth referred to as utilization.

- We compare the performance of omnidirectional and sector antennas setups using utilization, throughput, and packet success ratio (PSR) as the performance metrics. We consider the setup where $J$ sector antennas together cover the entire azimuth and the radiation patterns of the consecutive antennas are allowed to overlap.

\section{System ModeL}

Consider a vehicular ad hoc network where the receiver is surrounded by $N$ vehicles which are distributed according to a two-dimensional distribution. We assume that the $N$ vehicles are in each other's carrier sensing range and contend for the channel using the EDCA mechanism in the broadcast mode. Moreover, in our model the carrier sensing and packet transmission is performed in all directions. The probability of successfully decoding a packet at the receiver depends on the number of simultaneous transmissions and the wireless channel between the transmitters and the receiver. In the following subsections the system model used to derive the performance metrics is described.

\section{A. Notation}

The following notation is used in the paper. Lowercase letters, e.g., $x$ and lowercase boldface letters, e.g., $\mathbf{x}$ denote scalars and vectors, respectively. The sum of the elements in vector $\mathbf{x}$ is denoted by $|\mathbf{x}|$. Uppercase letters, e.g., $X$ and uppercase boldface letters, e.g., $\mathbf{X}$ denote random variables and random vectors, respectively. Sets are denoted by calligraphic letters, e.g., $\mathcal{X}$, with $|\mathcal{X}|$ denoting its cardinality. In the probabilities involving discrete random variables and vectors, the random variable is omitted to keep the notation compact, e.g., $\operatorname{Pr}\{m\} \triangleq \operatorname{Pr}\{M=m\}$ and $\operatorname{Pr}\{\mathbf{m}\} \triangleq \operatorname{Pr}\{\mathbf{M}=\mathbf{m}\}$. The probability density function (pdf) and cumulative density function (cdf) of a continuous random variable (RV) $X$ is denoted by $f_{X}(x)$ and $F_{X}(x)$, respectively. We denote the modulo addition and subtraction with respect to $J$ as $(j \pm k)_{J} \triangleq(j \pm k) \bmod J$. The indicator function $\mathbb{1}\{C\}=1$ if the condition $C$ is true and 0 otherwise. The differentials are written immediately after their corresponding integrals for improved readability, e.g.,

$$
z=\int_{a}^{b} \mathrm{~d} x \int_{c}^{d} \mathrm{~d} y f(x, y) .
$$

\section{B. Transmission Probability}

In the EDCA mechanism, a vehicle transmits a packet at the end of a channel busy slot or a channel idle slot depending on the state of its backoff and the availability of a packet to 
transmit. The number of simultaneous transmissions is typically analyzed by deriving the probability with which a vehicle transmits a packet at the end of a generic slot (a busy slot or an idle slot), referred to as the transmission probability. The transmission probabilities of messages belonging to different ACs of EDCA have been derived in [18]. Although the V2V broadcast messages belong to different ACs, majority of the messages consist of periodic cooperative awareness messages (CAMs). In this work, we assume that the vehicles broadcast periodic CAMs that belong to a single AC. The CAMs are generated periodically with a rate of $\lambda$ packets per second per vehicle. A packet rate of $\lambda=10$ has been recommended for the CAMs to support safety critical applications [19]. In this scenario with a small $\lambda$, referred to as the unsaturated buffer scenario, a vehicle might have to wait for a new CAM packet to be generated after the transmission of the current packet. The transmission probability in the unsaturated scenario is dependent on $\lambda$ and $N$, and has been studied in [5]. The authors of [5] model the packet arrival as a Poisson arrival process to include the unsaturated behavior in the discrete time Markov chain model that is used to derive the transmission probability. Furthermore, the transmission probability in [5] has been derived with the assumption that every vehicle is in each other's carrier sensing range and there are no hidden terminals. We adopt the transmission probability derived in [5], which is given by ${ }^{1}$

$$
\begin{aligned}
\tau_{N}= & {\left[\frac{W+1}{2}+\left(\frac{(1-\zeta) / W}{a_{\mathrm{b}} b+a_{\mathrm{i}}(1-b)}\right)\left(1+\frac{1-(1-a)^{W-1}}{a}\right)\right.} \\
& \left.\left(1+\frac{a_{\mathrm{b}} b(W-1)}{2}\right)-\frac{1-\zeta}{W} \frac{1-(1-a)^{W-1}}{a}\right]^{-1},
\end{aligned}
$$

where $W$ is the contention window size, $\zeta=\min \left\{1, \lambda T_{\text {ser }}\right\}$ is the probability that a packet is queued for transmission after the completion of the current transmission, $T_{\text {ser }}$ is the average service time, and $b=1-\left(1-\tau_{N}\right)^{N-1}$ is the channel busy probability experienced by a vehicle. The average service time is given by

$$
T_{\mathrm{ser}}=T_{\mathrm{b}}+\frac{b T_{\mathrm{b}}}{T_{\mathrm{avg}}}\left(\frac{T_{\mathrm{b}}}{2}+\frac{W-1}{2} T_{\mathrm{avg}}\right),
$$

where $T_{\mathrm{avg}}$ is the average slot duration, and $T_{\mathrm{b}}$ is the duration of a busy slot which is the same for both the successful and failed packet transmissions since acknowledgments are absent in the broadcast mode. The duration of a busy slot is given by [18]

$$
T_{\mathrm{b}}=T_{\mathrm{p}}+\mathrm{SIFS}+\operatorname{AIFSN}[\mathrm{AC}] \cdot T_{\mathrm{i}}+\delta,
$$

where $T_{\mathrm{p}}$ is the duration of the packets including headers, SIFS is the short interframe space, AIFSN $[A C]$ is the arbitration interframe space number of access class $\mathrm{AC}, T_{\mathrm{i}}$ is the duration of an idle slot and $\delta$ is the propagation delay. The average slot duration is $T_{\mathrm{avg}}=b T_{\mathrm{b}}+(1-b) T_{\mathrm{i}}$. The quantities $a=1-\exp \left(-\lambda T_{\mathrm{avg}}\right), a_{\mathrm{b}}=1-\exp \left(-\lambda T_{\mathrm{b}}\right)$, and $a_{\mathrm{i}}=$ $1-\exp \left(-\lambda T_{\mathrm{i}}\right)$ are the probabilities that a packet arrives during the generic slot, busy slot, and an idle slot, respectively. The

\footnotetext{
${ }^{1} \mathrm{~A}$ typo in the original equation is corrected.
}

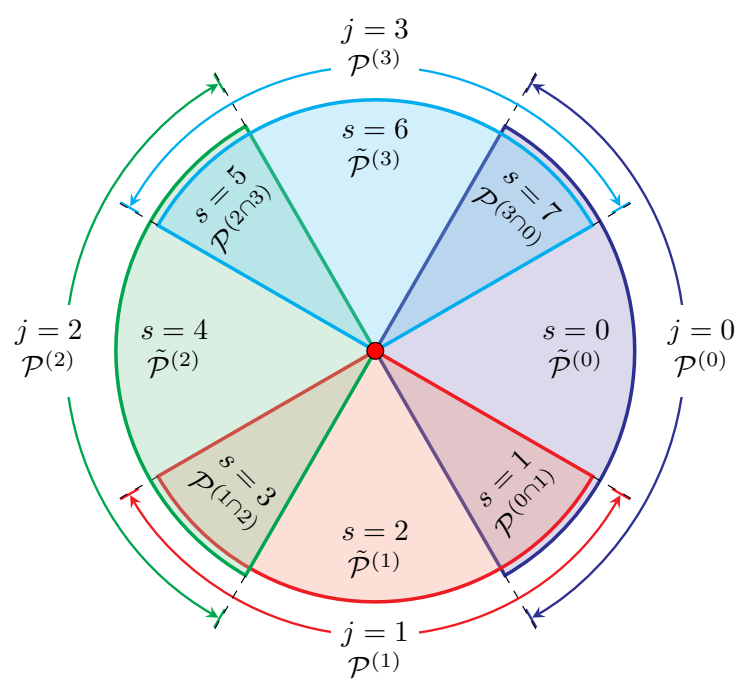

Fig. 1. The schematic diagram showing $J=4$ overlapping antennas that together cover the entire azimuth. The $J$ antennas, $S=2 J$ disjoint sectors, and the sets of received powers corresponding to the different antennas and sectors are illustrated.

transmission probability $\tau_{N}$ has been validated by the authors of [5] using network simulators OMNeT++ and MiXiM.

\section{Antenna and Receiver Configuration}

We consider a setup where the receiver is equipped with $J$ sector antennas. The $J$ sector antennas together cover the entire azimuth. We allow the radiation patterns of the adjacent antennas to partially overlap as shown in Fig. 1. We index the antennas with $j \in\{0,1, \ldots, J-1\}$. Antenna $j$ has a constant directive power gain of $G_{j}$. The angular range of Antenna $j$ is given by the interval $\left[\theta_{1}^{(j)}, \theta_{2}^{(j)}\right]$, and the beamwidth of the antenna, $\Theta^{(j)}$, is given by the length of the interval. Furthermore, we divide the azimuth into $S=2 J$ disjoint sectors indexed by $s \in\{0,1, \ldots, S-1\}$ so that the angular range of an antenna is comprised of three sectors among which two are shared with its adjacent antennas (see Fig. 1). The angular range of Sector $s$ is given by the interval $\left[\varphi_{1}^{(s)}, \varphi_{2}^{(s)}\right)$.

We assume a scenario where the transmitted signal from a transmitting vehicle arrives at the receiver in a narrow AOA. Therefore, a receiver antenna receives power only from the transmitters that are in its angular coverage region. In our setup, the receiver is capable of processing the output of each antenna separately to decode a packet. A packet is successfully decoded at an antenna when the decoding criterion discussed in Section II-D is satisfied. This allows the receiver to decode upto $J$ packets simultaneously. We derive the average number of packets successfully decoded in a generic slot in this setup, henceforth referred to as decoding success number, and compare it to the case of a single omnidirectional antenna.

The decoding success number depends on the number of transmitters in the coverage region of each antenna and hence on the angular distribution of the vehicles. The angular distribution can vary between vehicular scenarios; for example, the receiver is likely to have more vehicles towards its front and rear than to its sides in a highway scenario. To take this 
into account, we model the angular orientation of a vehicle with respect to the receiver as a RV $\Phi$ with pdf $f_{\Phi}(\phi)$. Consequently, the probability of a vehicle belonging to Sector $s$ is given by

$$
q_{s}=\int_{\varphi_{1}^{(s)}}^{\varphi_{2}^{(s)}} \mathrm{d} \phi f_{\Phi}(\phi)
$$

and we define the vector $\mathbf{q}=\left[q_{0}, q_{1}, \ldots, q_{S-1}\right]$ such that $|\mathbf{q}|=1$. To model the case of $J>1$ nonoverlapping antennas that cover the entire azimuth we retain the $S=2 J$ sector model and assign the overlapping sectors an angular range of 0 radians.

Let $n_{s}$ denote the number of vehicles in the coverage region of Sector $s$ and define the vector $\mathbf{n}=\left[n_{0}, n_{1}, \ldots, n_{S-1}\right]$ such that $|\mathbf{n}|=N$. The probability that the $N$ vehicles are distributed in $S$ sectors with $n_{s}$ vehicles in Sector $s$ is given by

$$
\operatorname{Pr}\{\mathbf{n}\}=\frac{|\mathbf{n}| !}{n_{0} ! n_{1} ! \ldots n_{S-1} !} \prod_{s=0}^{S-1} q_{s}^{n_{s}},
$$

where the first term is the multinomial coefficient which gives the number of ways of distributing the $N$ vehicles as $\mathbf{n}$.

Let $t_{s} \leq n_{s}$ denote the number of vehicles in Sector $s$ that transmit simultaneously at the end of a generic slot and define the vector $\mathbf{t}=\left[t_{0}, t_{1}, \ldots, t_{S-1}\right]$. The conditional probability of $t_{s}$ vehicles transmitting a packet simultaneously given that there are $n_{s}$ vehicles in Sector $s$ is given by

$$
\operatorname{Pr}\left\{t_{s} \mid n_{s}\right\}=\left(\begin{array}{c}
n_{s} \\
t_{s}
\end{array}\right) \tau_{N}^{t_{s}}\left(1-\tau_{N}\right)^{n_{s}-t_{s}}, 0 \leq t_{s} \leq n_{s} .
$$

Consequently,

$$
\operatorname{Pr}\{\mathbf{t} \mid \mathbf{n}\}=\prod_{s=0}^{S-1}\left(\begin{array}{c}
n_{s} \\
t_{s}
\end{array}\right) \tau_{N}^{t_{s}}\left(1-\tau_{N}\right)^{n_{s}-t_{s}}, 0 \leq t_{s} \leq n_{s} .
$$

Using (3) and (4), we have

$$
\begin{aligned}
\operatorname{Pr}\{\mathbf{t}\}= & \sum_{\forall \mathbf{n}} \operatorname{Pr}\{\mathbf{t} \mid \mathbf{n}\} \operatorname{Pr}\{\mathbf{n}\}, 0 \leq t_{s} \leq n_{s}, 0 \leq|\mathbf{t}| \leq N \\
= & \left(\begin{array}{c}
N \\
|\mathbf{t}|
\end{array}\right) \tau_{N}^{|\mathbf{t}|}\left(1-\tau_{N}\right)^{N-|\mathbf{t}|} \frac{|\mathbf{t}| !}{t_{0} ! t_{1} ! \ldots t_{S-1} !} \times \\
& \prod_{s=0}^{S-1} q_{s}^{t_{s}} \underbrace{\sum_{\mathbf{z} \in \mathcal{Z}} \frac{|\mathbf{z}| !}{z_{0} ! z_{1} ! \ldots z_{S-1} !} \prod_{s=0}^{S-1} q_{s}^{z_{s}}}_{=1}
\end{aligned}
$$

where $\mathcal{Z}=\left\{\mathbf{z}: z_{s}=n_{s}-t_{s}, n_{s} \geq t_{s}\right\}$. The sum over the set $\mathcal{Z}$ evaluates to 1 since it is the sum of probabilities of all possible realizations of $\mathbf{z}$

Let $m=|\mathbf{t}|$ denote the total number of vehicles transmitting simultaneously from all the sectors. We index the transmitting vehicles with $x \in\{1,2, \ldots, m\}$ and denote the power due to transmitter $x$ by $P_{x}$. Under the assumption that the powers are continuously distributed, the probability of two powers being equal is 0 . To avoid complicated notation, we assume that the received powers are all distinct.

Let $\mathcal{S}^{(j)}$ denote the set of sectors that are in the angular range of Antenna $j$. The number of transmitters in the angular range of Antenna $j$ is given by $m_{j}=\sum_{s \in \mathcal{S}^{(j)}} t_{s}$. Let $\mathcal{X}^{(j)}$ denote the set of $m_{j}$ transmitters that are in the coverage range of Antenna $j$. We define the following sets and quantities that are used in the rest of the paper.

$$
\begin{aligned}
\mathcal{P}^{(j)} & =\left\{P_{x}: x \in \mathcal{X}^{(j)}\right\}, m_{j}=\left|\mathcal{P}^{(j)}\right|, \\
\mathcal{P}^{(j \cap k)} & =\left\{P_{x}: x \in\left(\mathcal{X}^{(j)} \cap \mathcal{X}^{(k)}\right)\right\}, m_{j \cap k}=\left|\mathcal{P}^{(j \cap k)}\right|, \\
\mathcal{P}^{(j \cup k)} & =\left\{P_{x}: x \in\left(\mathcal{X}^{(j)} \cup \mathcal{X}^{(k)}\right)\right\}, m_{j \cup k}=\left|\mathcal{P}^{(j \cup k)}\right|, \\
\tilde{\mathcal{P}}^{(j)} & =\left\{P_{x}: x \in\left(\mathcal{X}^{(j)} \backslash\left(\mathcal{X}^{(j-1)_{J}} \cup \mathcal{X}^{(j+1)_{J}}\right)\right)\right\}, \\
\mathcal{N}^{(j)} & =\left\{(j-1)_{J},(j+1)_{J}\right\},
\end{aligned}
$$

where $\mathcal{P}^{(j)}$ is the set of powers received by Antenna $j$. The sets $\mathcal{P}^{(j \cap k)}$ and $\mathcal{P}^{(j \cup k)}$ are the sets of powers in the intersection and the union of Antennas $j$ and $k$, respectively; $\tilde{\mathcal{P}}^{(j)}$ is the set of powers received only by Antenna $j$ (see Fig. 1); and $\mathcal{N}^{(j)}$ denotes the set of neighbors of Antenna $j$. Note that for $J=2, \mathcal{P}^{(0 \cap 1)}$ and $\mathcal{P}^{(1 \cap 0)}$ denote two different sets.

\section{Packet Decoding Criterion}

In this section we study the criterion for successfully decoding a packet in the all-to-all broadcast scenario, where it is beneficial for the receiver to decode the packet with the largest received power. To achieve this in practice we assume that the receiver is constantly searching for a frame preamble even after having detected a frame; and when a preamble with a larger average power is detected, the receiver ignores the previously detected frame and processes the newly detected frame [20].

We model the $m_{j}$ powers in the set $\mathcal{P}^{(j)}$ as i.i.d RVs having a pdf $f_{P^{(j)}}(p)$, where $P^{(j)}$ is the RV corresponding to any one of the i.i.d received powers. Furthermore, we order the powers and denote the order statistics by

$$
P_{(1)}^{(j)} \geq P_{(2)}^{(j)} \geq \cdots \geq P_{\left(m_{j}\right)}^{(j)},
$$

where $P_{(l)}^{(j)}$ is the RV corresponding to the $l$ th largest power among the $m_{j}$ i.i.d powers at Antenna $j$. Note that the subscript with parenthesis is used for an ordered RV. Let

$$
P_{\mathrm{I}}^{(j)}=\sum_{l=2}^{m_{j}} P_{(l)}^{(j)}
$$

denote the interference power at Antenna $j$. Consequently, the SINR at Antenna $j$ with the directive power gain $G_{j}$ is given by

$$
\Gamma_{j}=\frac{G_{j} P_{(1)}^{(j)}}{G_{j} P_{\mathrm{I}}^{(j)}+\sigma^{2}}=\frac{P_{(1)}^{(j)}}{P_{\mathrm{I}}^{(j)}+\sigma_{j}^{2}},
$$

where $\sigma^{2}$ is the additive noise power and $\sigma_{j}^{2}=\sigma^{2} / G_{j}$. The SINR $\Gamma_{j}$ is a RV and we define the decoding criterion as $\Gamma_{j} \geq$ $\psi$, where $\psi$ is the minimum SINR required to successfully decode a packet. Note that the above SINR definition differs from the one used in unicast communications setup where the interfering powers are independent of the power of the desired packet. 
In the case of a single transmitter, i.e., $m_{j}=1$, we have $P_{\mathrm{I}}^{(j)}=0, P_{(1)}^{(j)}=P^{(j)}$, and the SINR reduces to signalto-noise power ratio (SNR). The probability of successfully decoding a packet is then given by

$$
\begin{aligned}
p_{\mathrm{D}}^{(j)}= & \operatorname{Pr}\left\{\Gamma_{j} \geq \psi\right\} \\
= & \begin{cases}0 & \text { for } m_{j}=0, \\
\operatorname{Pr}\left\{\frac{P^{(j)}}{\sigma_{j}^{2}} \geq \psi\right\}=1-F_{P^{(j)}}\left(\psi \sigma_{j}^{2}\right) & \text { for } m_{j}=1, \\
\operatorname{Pr}\left\{\frac{P_{(1)}^{(j)}}{P_{\mathrm{I}}^{(j)}+\sigma_{j}^{2}} \geq \psi\right\} & \text { for } m_{j} \geq 2 .\end{cases}
\end{aligned}
$$

Theorem 1 . The probability of decoding when $m_{j} \geq 2$ is given by

$$
\begin{aligned}
p_{\mathrm{D}}^{(j)} & =\operatorname{Pr}\left\{\frac{P_{(1)}^{(j)}}{P_{\mathrm{I}}^{(j)}+\sigma_{j}^{2}} \geq \psi\right\} \\
& =\int_{0}^{\infty} \mathrm{d} u \int_{0}^{\frac{u-\psi \sigma_{j}^{2}}{\psi}} \mathrm{d} v f_{P_{(1)}^{(j)}, P_{\mathrm{I}}^{(j)}}(u, v),
\end{aligned}
$$

where $f_{P_{(1)}^{(j)}, P_{\mathrm{I}}^{(j)}}(u, v)$ is the joint distribution of the largest and the sum of the remaining of $m_{j}$ i.i.d RVs.

Proof: See Appendix A.

The joint probability in (9) is given by [21], [22]

$$
\begin{aligned}
& f_{P_{(1)}^{(j)}, P_{\mathrm{I}}^{(j)}}(u, v)=m_{j} f_{P^{(j)}}(u) \times \\
& \mathcal{L}_{s}^{-1}\left\{\left(\int_{0}^{u} \mathrm{~d} x f_{P^{(j)}}(x) \exp (-x s)\right)^{m_{j}-1}\right\}(v), u \geq \frac{v}{m_{j}-1},
\end{aligned}
$$

where $P^{(j)}$ is the RV corresponding to any one of the i.i.d received powers and $\mathcal{L}_{s}^{-1}\{\cdot\}(v)$ is the inverse Laplace transform from $s$ to $v$. The joint probability involving inverse Laplace transform may be analytically intractable for an arbitrary distribution $f_{P(j)}(p)$. Therefore, we present an upper and lower bound to (9). For $m_{j} \geq 2$, the interference term in (9) can be lower and upper bounded as

$$
P_{(2)}^{(j)} \leq P_{\mathrm{I}}^{(j)} \leq\left(m_{j}-1\right) P_{(2)}^{(j)} .
$$

The lower bound is found by replacing the summation in (7) by its largest term $P_{(2)}^{(j)}$ and the upper bound is found by replacing each term in the summation by its largest term $P_{(2)}^{(j)}$.

Let

$$
\tilde{p}_{\mathrm{D}}\left(m, \sigma^{2}, c, l\right)= \begin{cases}0 & \text { for } m=0, \\ 1-F_{P}\left(\psi \sigma^{2}\right) & \text { for } m=1, \\ \operatorname{Pr}\left\{\frac{P_{(1)}}{c P_{(l)}+\sigma^{2}} \geq \psi\right\} & \text { for } m \geq 2,\end{cases}
$$

where $P_{(l)}$ is the RV corresponding to the $l$ th largest power among the $m$ i.i.d received powers having a pdf $f_{P}(p)$ and $P$ is the RV corresponding to any one of the i.i.d received powers. When $m \geq 2$ and $c=0$, the probability in (12) is given by

$$
\begin{aligned}
\tilde{p}_{\mathrm{D}}\left(m, \sigma^{2}, 0, l\right) & =\operatorname{Pr}\left\{P_{(1)} \geq \sigma^{2} \psi\right\} \\
& =1-F_{P_{(1)}}\left(\sigma^{2} \psi\right)=1-\left(F_{P}\left(\sigma^{2} \psi\right)\right)^{m}
\end{aligned}
$$

where $F_{P_{(1)}}(p)=\left(F_{P}(p)\right)^{m}$ is the cdf of the largest ordered RV among $m$ i.i.d RVs [23]. Using the result in Appendix A, the probability in (12) when $m \geq 2$ and $c>0$ is given by

$$
\tilde{p}_{\mathrm{D}}\left(m, \sigma^{2}, c, l\right)=\int_{0}^{\infty} \mathrm{d} u \int_{0}^{\frac{u-\psi \sigma^{2}}{c \psi}} \mathrm{d} v f_{P_{(1)}, P_{(l)}}(u, v) .
$$

The joint probability of the largest and the lth largest among $m$ i.i.d RVs for $l \geq 2$ is given by [23]

$$
\begin{aligned}
& f_{P_{(1)}, P_{(l)}}(u, v)=\frac{m !}{(l-2) !(m-l) !} f_{P}(u) \times \\
& {\left[F_{P}(u)-F_{P}(v)\right]^{l-2} f_{P}(v)\left[F_{P}(v)\right]^{m-l}, u \geq v .}
\end{aligned}
$$

The joint probability is obtained by using only the pdf and the cdf of the unordered RV $P$ corresponding to any one of the $m$ i.i.d received powers.

Using the definition in (12) with $l=2$ and the inequalities in (11), the probability of decoding when $m_{j} \geq 2$ can be bounded as

$$
\tilde{p}_{\mathrm{D}}\left(m_{j}, \sigma_{j}^{2}, m_{j}-1,2\right) \leq p_{\mathrm{D}}^{(j)} \leq \tilde{p}_{\mathrm{D}}\left(m_{j}, \sigma_{j}^{2}, 1,2\right) .
$$

\section{E. Decoding Success Number}

In the case of $J$ overlapping antennas, packets from transmitters in an overlapping sector are received by the two adjacent antennas that contain the sector (see Fig. 1). Such packets may be successfully decoded at both the antennas if the decoding criterion is met at both the antennas; this results in only one packet being decoded and must be taken into account when computing the decoding success number. As a result, the decoding success number in this scenario cannot be calculated by adding the decoding success numbers from each antenna. The decoding success number is given by

$$
\bar{D}(N)=\sum_{\forall \mathbf{t}} \operatorname{Pr}\{\mathbf{t}\} D(\mathbf{t}),
$$

where $D(\mathbf{t})$ is the average number of unique packets that are successfully decoded when $\mathbf{t}$ vehicles are transmitting simultaneously. Using (5) in the above expression we have

$\bar{D}(N)=\sum_{\forall \mathbf{t}}\left(\begin{array}{l}N \\ |\mathbf{t}|\end{array}\right) \tau_{N}^{|\mathbf{t}|}\left(1-\tau_{N}\right)^{N-|\mathbf{t}|} \frac{|\mathbf{t}| !}{t_{0} ! t_{1} ! \ldots t_{S-1} !} \prod_{s=0}^{S-1} q_{s}^{t_{s}} D(\mathbf{t})$
$=\sum_{m=1}^{N}\left(\begin{array}{l}N \\ m\end{array}\right) \frac{\tau_{N}^{m}}{\left(1-\tau_{N}\right)^{m-N}}\left(\sum_{\mathbf{t} \in \mathcal{Y}_{m}} \frac{|\mathbf{t}| !}{t_{0} ! t_{1} ! \ldots t_{S-1} !} \prod_{s=0}^{S-1} q_{s}^{t_{s}} D(\mathbf{t})\right)$,

where the sum in the first equality is split into two sums in the second equality and the set $\mathcal{Y}_{m}=\{\mathbf{t}:|\mathbf{t}|=m\}$.

In the case of a single omnidirectional antenna, $J=1$, $S=1$, and $q_{1}=1$. Therefore, the decoding success number in this case simplifies to

$$
\bar{D}_{\mathrm{O}}(N)=\sum_{m_{\mathrm{O}}=1}^{N}\left(\begin{array}{c}
N \\
m_{\mathrm{O}}
\end{array}\right) \tau_{N}^{m_{\mathrm{O}}}\left(1-\tau_{N}\right)^{N-m_{\mathrm{O}}} \operatorname{Pr}\left\{\Gamma_{\mathrm{O}} \geq \psi\right\}
$$

where $\Gamma_{\mathrm{O}}$ is the SINR at the omnidirectional antenna due to $m=m_{\mathrm{O}}$ transmitters. 
Theorem 2. For $J \geq 2, D(\mathbf{t})$ is given by

$$
\begin{aligned}
D(\mathbf{t}) & =\sum_{j=0}^{J-1} \operatorname{Pr}\left\{\frac{P_{(1)}^{(j)}}{P_{\mathrm{I}}^{(j)}+\sigma_{j}^{2}} \geq \psi\right\}-\sum_{(j, k) \in \mathcal{O}}\left(\mathbb{1}\left\{m_{j \cap k}>0\right\}\right. \\
\operatorname{Pr} & \left.\left\{\frac{P_{(1)}^{(j \cup k)}}{P_{\mathrm{I}}^{(j)}+\sigma_{j}^{2}} \geq \psi, \frac{P_{(1)}^{(j \cup k)}}{P_{\mathrm{I}}^{(k)}+\sigma_{k}^{2}} \geq \psi\right\} \frac{m_{j \cap k}}{m_{j \cup k}}\right),
\end{aligned}
$$

where the set $\mathcal{O}=\left\{\left(j,(j+1)_{J}\right): j=0,1, \ldots, J-1\right\}$ and $P_{(1)}^{(j \cup k)}$ is the largest power in $\mathcal{P}^{(j \cup k)}$.

Proof: See Appendix B.

The decoding success number for the overlapping antenna setup is obtained by using (18) in (16). In the special case of nonoverlapping antennas, the number of transmitters in the overlapping regions $m_{j \cap k}=0$. Computing the joint probability term in (18) analytically is nontrivial as explained in Appendix B. Therefore, we provide bounds to $D(\mathbf{t})$ that are easier to compute.

Theorem 3. $D(\mathbf{t})$ in Theorem 2 is lower and upper bounded as

$$
\begin{gathered}
D_{\mathrm{LB}}(\mathbf{t})=\sum_{j=0}^{J-1} \tilde{p}_{\mathrm{D}}\left(m_{j}, \sigma_{j}^{2}, m_{j}-1,2\right)-\sum_{(j, k) \in \mathcal{O}}\left(\mathbb{1}\left\{m_{j \cap k}>0\right\}\right. \\
\left.\tilde{p}_{\mathrm{D}}\left(m_{j \cup k},\left(\sigma_{j, k}^{-}\right)^{2}, c_{j, k}, l_{j, k}\right) \frac{m_{j \cap k}}{m_{j \cup k}}\right), \\
D_{\mathrm{UB}}(\mathbf{t})=\sum_{j=0}^{J-1} \tilde{p}_{\mathrm{D}}\left(m_{j}, \sigma_{j}^{2}, 1,2\right)-\sum_{(j, k) \in \mathcal{O}}\left(\mathbb{1}\left\{m_{j \cap k}>0\right\}\right. \\
\left.\tilde{p}_{\mathrm{D}}\left(m_{j \cup k},\left(\sigma_{j, k}^{+}\right)^{2}, m_{j, k}^{+}-1,2\right) \frac{m_{j \cap k}}{m_{j \cup k}}\right),
\end{gathered}
$$

respectively. And the quantities $m_{j, k}^{+}=\max \left\{m_{j}, m_{k}\right\}$, $m_{j, k}^{-}=\min \left\{m_{j}, m_{k}\right\}, \sigma_{j, k}^{+}=\max \left\{\sigma_{j}, \sigma_{k}\right\}, \sigma_{j, k}^{-}=$ $\min \left\{\sigma_{j}, \sigma_{k}\right\}$; and $c_{j, k}=\mathbb{1}\left\{m_{j, k}^{-} \neq 1\right\}$ and $l_{j, k}=m_{j \cup k}-$ $m_{j, k}^{-}+2$.

\section{Proof: See Appendix C.}

Corollary 1. In the interference limited scenario, where the noise power is smaller than any of the received powers, an approximation to the lower bound $D_{\mathrm{LB}}(\mathbf{t})$ in Theorem 3 is given by

$$
\begin{gathered}
\hat{D}_{\mathrm{LB}}(\mathbf{t})=\sum_{j=0}^{J-1} \tilde{p}_{\mathrm{D}}\left(m_{j}, \sigma_{j}^{2}, m_{j}-1,2\right)-\sum_{(j, k) \in \mathcal{O}}\left(\mathbb{1}\left\{m_{j \cap k}>0\right\}\right. \\
\left.\tilde{p}_{\mathrm{D}}\left(m_{j \cup k},\left(\sigma_{j, k}^{-}\right)^{2}, 1, \hat{l}_{j, k}\right) \frac{m_{j \cap k}}{m_{j \cup k}}\right),
\end{gathered}
$$

where $\hat{l}_{j, k}=2 \cdot \mathbb{1}\left\{m_{j, k}^{-}=1\right\}+\left(m_{j \cup k}-m_{j, k}^{-}+2\right)$. $\mathbb{1}\left\{m_{j, k}^{-} \neq 1\right\}$.

Proof: See Appendix C.

The bounds (19), (20), and the approximation (21) can be computed using $\tilde{p}_{\mathrm{D}}\left(m, \sigma^{2}, c, l\right)$ derived in Section II-D. Using the results (19), (20), and (21) in (16) we obtain the bounds and approximation to the decoding success number.

\section{F. Performance Metrics}

We use normalized utilization, throughput, and PSR as the performance metrics to compare the performance of the different antenna configurations. Normalized utilization, $U(N)$, defined as the average number of packets successfully decoded per packet duration is given by [18], [24]

$$
U(N)=\bar{D}(N) T_{\mathrm{p}} / T_{\mathrm{g}}(N),
$$

where $\bar{D}(N)$ is the decoding success number derived for the generic slot duration $T_{\mathrm{g}}(N)=\left(1-b^{\star}\right) T_{\mathrm{i}}+b^{\star} T_{\mathrm{b}}$ where $b^{\star}=$ $1-\left(1-\tau_{N}\right)^{N}$ is the probability that at least one vehicle transmits. Note that $U(N)$ can be larger than one for the case of multiple sector antennas as $\bar{D}(N)$ can be larger than one. In the case of a single omnidirectional antenna, $U(N)$ can also be interpreted as the fraction of the total time used for successful transmissions [24]. Throughput $X(N)$, defined as the average number of successful packets decoded per second, is given by

$$
X(N)=\bar{D}(N) / T_{\mathrm{g}}(N)=U(N) / T_{\mathrm{p}} .
$$

Note that the throughput is a scaled version of the utilization. Also, the PSR is given by

$$
\operatorname{PSR}(N)=\bar{D}(N) /\left(N \tau_{N}\right) .
$$

\section{VEHICLES UNIFORMLY DISTRIBUTED IN AN ANNULAR SECTOR}

In this section, we apply the framework developed in Section II to the case when the vehicles are uniformly distributed in an annular sector with the receiver at the origin. Let $R_{\mathrm{O}}$ and $R_{\mathrm{I}}$ denote the outer and the inner radius of the annular sector $s$, respectively, and let $\left[\varphi_{1}^{(s)}, \varphi_{1}^{(s)}+\Delta_{\varphi^{(s)}}\right)$ denote the angular range. We assume that the sector is nonempty, i.e., that $R_{\mathrm{O}}>R_{\mathrm{I}}$ and $0<\Delta_{\varphi(s)}<2 \pi$. The outer radius $R_{\mathrm{O}}$ is chosen according to the CSMA sensing range. The inner radius $R_{\mathrm{I}}>0$ is used to avoid transmitting vehicles being located too close to the receiver and for the path-loss model (PLM) to be valid. The distance between a transmitter in the annular sector and the receiver at the origin is modeled as a RV $R$. The cdf $F_{R}(r)$, which is the probability that a transmitter falls inside the annular sector with outer radius $r \leq R_{\mathrm{O}}$ is given by [25]

$$
F_{R}(r)=\frac{\left(r^{2}-R_{\mathrm{I}}^{2}\right)}{\left(R_{\mathrm{O}}^{2}-R_{\mathrm{I}}^{2}\right)}, \quad R_{\mathrm{I}} \leq r \leq R_{\mathrm{O}} .
$$

Subsequently, the pdf $f_{R}(r)$ of the distance $R$ is given by

$$
f_{R}(r)=\frac{\mathrm{d} F_{R}(r)}{\mathrm{d} r}=\frac{2 r}{R_{\mathrm{O}}^{2}-R_{\mathrm{I}}^{2}}, \quad R_{\mathrm{I}} \leq r \leq R_{\mathrm{O}} .
$$

The received power $p$ at the receiver due to a transmitter is modeled using a single slope PLM and is given by

$$
p=P_{\mathrm{T}} K_{\mathrm{I}}\left(r / R_{\mathrm{I}}\right)^{-\alpha}, \quad r>R_{\mathrm{I}}
$$

where $P_{T}$ is the constant transmit power, $K_{\mathrm{I}}$ is the gain at the reference distance $R_{\mathrm{I}}, r$ is the distance between the transmitter and the receiver, and $\alpha>1$ is the path-loss exponent. The received power can be further simplified as

$$
p=h(r)=\rho r^{-\alpha}, \quad R_{\mathrm{I}} \leq r \leq R_{\mathrm{O}},
$$


where $\rho=P_{\mathrm{T}} K_{\mathrm{I}} R_{\mathrm{I}}^{\alpha}$. The received power $P$ is a RV that is a monotonically decreasing function of $R$ and its pdf is given by

$$
\begin{aligned}
f_{P}(p) & =\left.\frac{f_{R}(r)}{\left|\frac{d p}{d r}\right|}\right|_{r=h^{-1}(p)} \\
& =\left.\frac{2 r}{\left(R_{\mathrm{O}}^{2}-R_{\mathrm{I}}^{2}\right)} \cdot \frac{1}{\rho \alpha r^{-(\alpha+1)}}\right|_{r=h^{-1}(p)} \\
& =\left.\frac{2 r^{\alpha+2}}{\left(R_{\mathrm{O}}^{2}-R_{\mathrm{I}}^{2}\right) \rho \alpha}\right|_{r=p^{-1 / \alpha} \rho^{1 / \alpha}} \\
& = \begin{cases}\beta p^{-(1+2 / \alpha)} & \text { for } \mathrm{p}_{1} \leq p \leq \mathrm{p}_{2}, \\
0 & \text { otherwise, },\end{cases}
\end{aligned}
$$

where

$$
\beta=\frac{2 \rho^{2 / \alpha}}{\left(R_{\mathrm{O}}^{2}-R_{\mathrm{I}}^{2}\right) \alpha}, \mathrm{p}_{1}=\rho R_{\mathrm{O}}^{-\alpha}, \text { and } \mathrm{p}_{2}=\rho R_{\mathrm{I}}^{-\alpha} .
$$

Subsequently, the cdf of the received power is given by

$$
\begin{aligned}
& F_{P}(p)=\int_{\mathrm{p}_{1}}^{p} \mathrm{~d} t \beta t^{-(1+2 / \alpha)}
\end{aligned}
$$

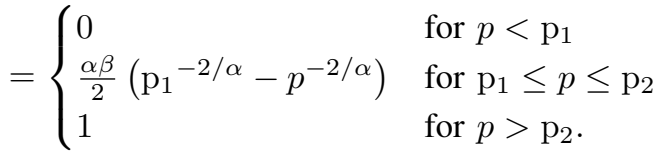

The received powers in an annular sector are modeled as i.i.d RVs with pdf $f_{P}(p)$ in (26). We use $2 J$ nonoverlapping annular sectors with the receiver at their origin to distribute the vehicles. Since the distribution of the powers within an annular sector is independent of the AOA, we first use the angular distribution $f_{\Phi}(\phi)$ to obtain $\mathbf{q}$, where $q_{s}$ is the probability that a transmitting vehicle belongs to Sector $s$. Using this approach we ignore the angular distribution within an sector while capturing the distribution of vehicles across the sectors.

\section{A. Decoding Success Number}

In this section we use the power distribution in the annular sector model to compute the decoding success number. The quantity $D(\mathbf{t})$ required to compute the decoding success number is given by (18). The probability $\operatorname{Pr}\left\{\Gamma_{j} \geq \psi\right\}$ in (18) requires the joint distribution $f_{P_{(1)}^{(j)}, P_{\mathrm{I}}^{(j)}}(u, v)$. Substituting the power distribution given by (26) in (10) we have

$$
\begin{aligned}
& f_{P_{(1)}^{(j)}, P_{\mathrm{I}}^{(j)}}(u, v)=m_{j} \beta u^{-(1+2 / \alpha)} \times \\
& \mathcal{L}_{s}^{-1}\left\{\left(\int_{\mathrm{p}_{1}}^{u} \mathrm{~d} x \beta x^{-\left(1+\frac{2}{\alpha}\right)} \exp (-x s)\right)^{m_{j}-1}\right\}(v), u \geq \frac{v}{m_{j}-1} .
\end{aligned}
$$

The integral in the above expression is an incomplete gamma function and the joint distribution is analytically intractable. Furthermore, computing the joint probability term in (18) analytically is nontrivial as explained in Appendix B. We therefore compute the bounds and the approximation to $D(\mathbf{t})$ given by (19), (20), and (21). The bounds require the computation of $\tilde{p}_{\mathrm{D}}\left(m, \sigma^{2}, c, l\right)$ defined in (12).
Theorem 4. For $m \geq 2$ and $c>0, \tilde{p}_{\mathrm{D}}\left(m, \sigma^{2}, c, l\right)$ is given by

$$
\begin{aligned}
& \tilde{p}_{\mathrm{D}}\left(m, \sigma^{2}, c, l\right)=\operatorname{Pr}\left\{\frac{P_{(1)}}{c P_{(l)}+\sigma^{2}} \geq \psi\right\} \\
& =\frac{m ! \beta^{m}(\alpha / 2)^{m-1}}{(l-2) !(m-l) !} \sum_{q=0}^{l-2} \sum_{r=0}^{m-l}\left(\begin{array}{c}
l-2 \\
q
\end{array}\right)\left(\begin{array}{c}
m-l \\
r
\end{array}\right) \mathrm{p}_{1}-\frac{2}{\alpha} r \\
& \frac{(-1)^{m+q-l-r}}{(m-r-q-1)}\left[\frac{\alpha \mathrm{p}_{1}-\frac{2}{\alpha}(m-r-q-1)}{2(q+1)}\left(\mathrm{p}_{0}^{-\frac{2}{\alpha}(q+1)}-\mathrm{p}_{2}{ }^{-\frac{2}{\alpha}(q+1)}\right)-\right. \\
& \left.(c \psi)^{\frac{2}{\alpha}(m-r-q-1)} \int_{\mathrm{p}_{0}}^{\mathrm{p}_{2}} \mathrm{~d} u u^{-\frac{2}{\alpha}(q+1)-1}\left(u-\psi \sigma^{2}\right)^{-\frac{2}{\alpha}(m-r-q-1)}\right],
\end{aligned}
$$

where $\mathrm{p}_{0}=c \psi \mathrm{p}_{1}+\psi \sigma^{2}$.

Proof: See Appendix D for the proof. For the special case of $\alpha=2$ and $l=2$, the probability $\tilde{p}_{\mathrm{D}}\left(m, \sigma^{2}, c, l\right)$ can be expressed using the hypergeometric function as shown in the appendix.

The integral in Theorem 4 cannot be simplified further for an arbitrary $\alpha$ due to the negative noninteger power $-2 / \alpha$ and can be evaluated numerically. However, in the interference limited scenario when the interference power is much larger than the noise power, the noise power $\sigma^{2}$ can be neglected. This assumption is reasonable in our annular ring geometry with path-loss power model where the smallest interference power $\mathrm{p}_{1}$ is much larger than the noise power. In the interference limited scenario, we assume that $\sigma^{2}=0$ and approximations of (19), (20), and (21) are obtained by using $\tilde{p}_{\mathrm{D}}(m, 0, c, l)$ instead of $\tilde{p}_{\mathrm{D}}\left(m, \sigma^{2}, c, l\right)$.

Theorem 5. For $m \geq 2$ and $c>0, \tilde{p}_{\mathrm{D}}(m, 0, c, l)$ is given by

$$
\begin{aligned}
& \tilde{p}_{\mathrm{D}}(m, 0, c, l)=\operatorname{Pr}\left\{\frac{P_{(1)}}{c P_{(l)}} \geq \psi\right\} \\
& =\frac{m !\left((\alpha \beta / 2) \mathrm{p}_{1}-\frac{2}{\alpha}\right)^{m}}{(l-2) !(m-l) !} \sum_{q=0}^{l-2} \sum_{r=0}^{m-l}\left(\begin{array}{c}
l-2 \\
q
\end{array}\right)\left(\begin{array}{c}
m-l \\
r
\end{array}\right) \times \\
& \frac{(-1)^{m+q-l-r}(c \psi)^{-\frac{2}{\alpha}(q+1)}}{m-r-q-1}\left[\frac{1-\nu^{q+1}}{q+1}-\frac{1-\nu^{m-r}}{m-r}\right],
\end{aligned}
$$

where $\nu=\left(\mathrm{p}_{2} /\left(c \psi \mathrm{p}_{1}\right)\right)^{-2 / \alpha}$.

Proof: See Appendix E for the proof.

\section{NUMERICAL RESUlts}

Utilization, throughput, and PSR metrics discussed in Section II-F are used to compare the performance of the different antenna setups in the annular ring model. The expressions derived in (17) and (18) can be used to compute the performance metrics for a generic channel model and antenna gain pattern by generating realizations of received powers and performing Monte Carlo simulations, with the only constraint being that the signal corresponding to a packet arrives within a single nonoverlapping sector. In this section, we consider a scenario where the performance metrics can be analyzed using the bounds derived in Section II.

We consider an annular geometry with inner and outer radius of $R_{\mathrm{I}}=10 \mathrm{~m}$ and $R_{\mathrm{O}}=400 \mathrm{~m}$, respectively. The distribution of the vehicles in different sectors in the case 
of the $J=4$ nonoverlapping antenna (NO) and the $J=4$ overlapping antenna (OA) setups denoted by $\mathbf{q}_{\mathrm{NO}}$ and qoA, are listed in Table I. The vectors $\mathbf{q}_{\mathrm{NO}}$ and $\mathbf{q}_{\mathrm{OA}}$ are arbitrarily chosen to suit a multilane highway scenario under the assumption that the receiver encounters a larger number of vehicles towards its front and rear directions in comparison to the sides and that the region of overlap between consecutive antennas is not large. The vectors have to be derived from the angular distribution of vehicles with respect to the receiver for the traffic scenario under consideration and the antenna radiation patterns, but this is outside the scope of this work.

A constant antenna gain of $G_{\mathrm{O}}=2.15 \mathrm{dBi}$ which is the maximum gain of a lossless half-wave dipole antenna in the azimuth plane is chosen for the omnidirectional antenna. A sector antenna with a constant gain used in our system model does not exist in reality. However, an antenna with a narrower beamwidth typically has a larger directional gain. Therefore, we obtain the constant gains of the $J=4$ sector antennas in the NO and OA setups by scaling $G_{\mathrm{O}}$ with respect to the beamwidth of the antennas, i.e., $G_{j}=G_{\mathrm{O}}\left(2 \pi / \Theta^{(j)}\right)$, where $\Theta^{(j)}$ is the beamwidth of constant gain antennas. The beamwidths for the NO and the OA setups are chosen as $\Theta_{\mathrm{NO}}^{(j)}=\pi / 2$ and $\Theta_{\mathrm{OA}}^{(j)}=2 \pi / 3$, respectively. Note that an angular dependent directive gain instead of a constant gain can be used to obtain the realizations of received powers and the performance metrics can be computed numerically by Monte Carlo simulations of (17) and (18). We have chosen to use constant antenna gains in this section to validate the analytic bounds derived in Section II-E.

We use the PLM parameters derived for the LOS and obstructed LOS scenarios in [6]. We restrict ourselves to the single slope model to be compatible with the proposed framework and hence use a single slope from the models described in [6]. The parameters of the two PLMs considered are listed in Table I. All the transmitters transmit over a bandwidth of $10 \mathrm{MHz}$ with a constant transmit power of $P_{\mathrm{T}}=33 \mathrm{dBm}$ and the noise power over the considered bandwidth is fixed at $\sigma^{2}=-104 \mathrm{dBm}$. A packet length of 400 bytes is considered and the packets are transmitted with a rate of $R_{\mathrm{b}}=6 \mathrm{Mb} / \mathrm{s}$, which is the default transmission rate for the CAM packets using the IEEE 802.11p standard. For the chosen packet length and the transmission rate, the threshold $\psi$ used in the decoding criterion depends on the channel, desired reliability, and the channel estimation scheme used at the receiver. We use a threshold of $\psi=10 \mathrm{~dB}$ for the results discussed in this section (see [26] for the influence of different channels and the channel estimation schemes on the threshold). The MAC parameters AIFSN and $W$, listed in Table I, used to derive the transmission probability $\tau_{N}$ correspond to the AC assigned to CAMs [27, Table 5].

The probability that a vehicle transmits at a generic slot boundary, $\tau_{N}$ in (1), for different $\lambda$ is shown in Fig. 2. As seen in the figure, $\tau_{N}$ reaches the transmission probability in the saturated scenario $(\zeta=1)$ as $\lambda$ increases. Note that the transmission probabilities in the figure correspond to the MAC parameters listed in Table I. For the remaining results described in this section, $\tau_{N}$ that corresponds to the packet arrival rate of $\lambda=10 \mathrm{pkt} / \mathrm{s}$ is used, which is the rate specified
TABLE I

Parameters in the ANNUlar Ring Model

\begin{tabular}{|c|c|}
\hline Parameters & Values \\
\hline$R_{\mathrm{O}}$ & $100 \mathrm{~m}$ \\
$R_{\mathrm{I}}$ & $10 \mathrm{~m}$ \\
$\mathbf{q}_{\mathrm{NO}}$ & {$[0.3,0,0.2,0,0.3,0,0.2,0]$} \\
qOA $_{\mathrm{A}}$ & {$[0.2,0.1,0.1,0.2,0.1,0.1,0.1]$} \\
$G_{\mathrm{O}}$ & $2.15 \mathrm{dBi}$ \\
$G_{j, \mathrm{NO}}, \forall j$ & $8.17 \mathrm{dBi}$ \\
$G_{j, \mathrm{OA}}, \forall j$ & $6.92 \mathrm{dBi}$ \\
$P_{\mathrm{T}}$ & $33 \mathrm{dBm}$ \\
$\sigma^{2}$ & $-104 \mathrm{dBm}$ \\
LEN & $3200 \mathrm{bit}$ \\
$R_{\mathrm{b}}$ & $6 \mathrm{Mb} / \mathrm{s}$ \\
$T_{\mathrm{p}}$ & LEN $/ R_{\mathrm{b}}$ \\
$\psi$ & $10 \mathrm{~dB}$ \\
$\lambda$ & $10 \mathrm{pkt} / \mathrm{s}$ \\
$T_{\mathrm{i}}$ & $13 \mu \mathrm{s}$ \\
$\delta$ & $2 \mu \mathrm{s}$ \\
SIFS & $32 \mu \mathrm{s}$ \\
AIFSN $[$ AC $]$ & 6 \\
$W$ & 16 \\
\hline & $\alpha_{1}=3.18$ \\
PLM 1 & $K_{\mathrm{I} 1}=-76.1 \mathrm{~dB}$ \\
& $\rho_{1}=-11.3 \mathrm{dBm}$ \\
\hline & $\alpha_{2}=1.66$ \\
PLM 2 & $K_{\mathrm{I} 2}=-66.1 \mathrm{~dB}$ \\
& $\rho_{2}=-16.5 \mathrm{dBm}$ \\
\hline
\end{tabular}

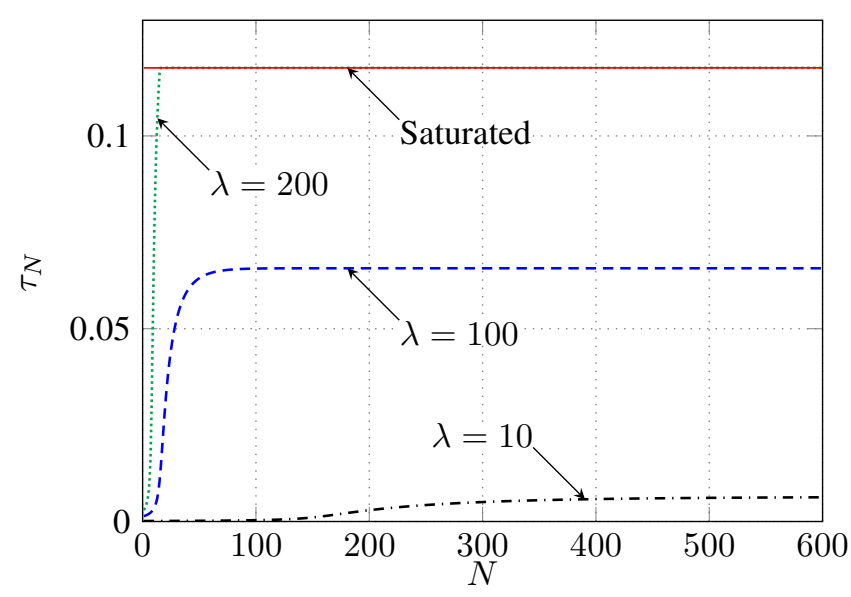

Fig. 2. Transmission probability as a function of $N$ for different packet generation rates $\lambda$.

for safety critical applications [19, Table 1].

In Fig. 3, utilization as a function of $N$ in the omnidirectional antenna setup is shown. The utilization using the ordered powers SINR model considered in this paper is shown for PLM 1 and PLM 2 models, indicated by PLM 1 and PLM 2, respectively. The results are obtained using the transmission probability $\tau_{N}$ and Monte Carlo simulations of (17). The figure also shows the utilization computed using the collision model where two simultaneous packet transmissions results in a packet decoding failure. The decoding success number in the case of the collision model for both path loss models is given by $\bar{D}_{\text {coll }}(N)=N \tau_{N}\left(1-\tau_{N}\right)^{N-1}$ [5]. Therefore, the utilization in the collision model depends only on the transmission probability and the number of vehicles, and is independent of the path loss models used to model the received powers. When the number of vehicles increases, resulting in higher 


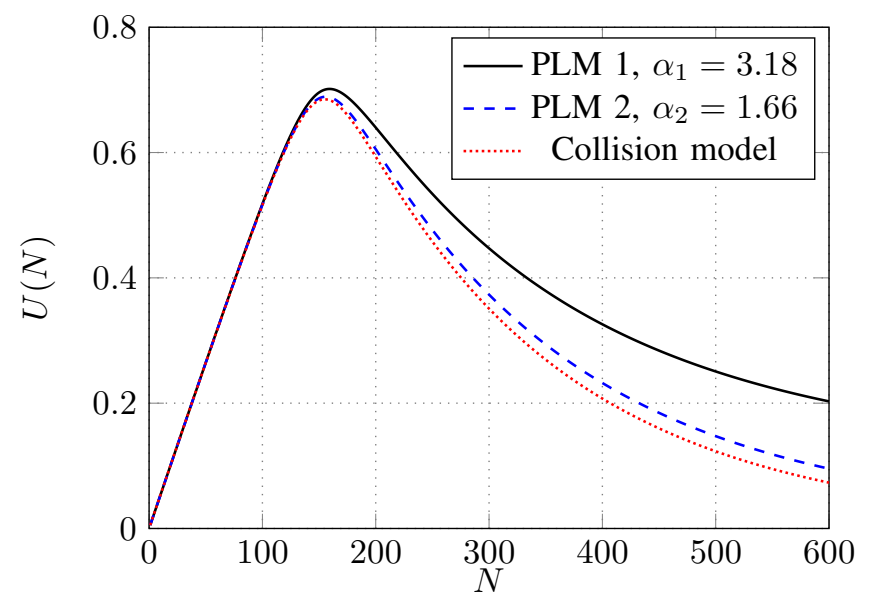

Fig. 3. Utilization in the omnidirectional antenna setup for different decoding criteria and pathloss models for $\lambda=10$.

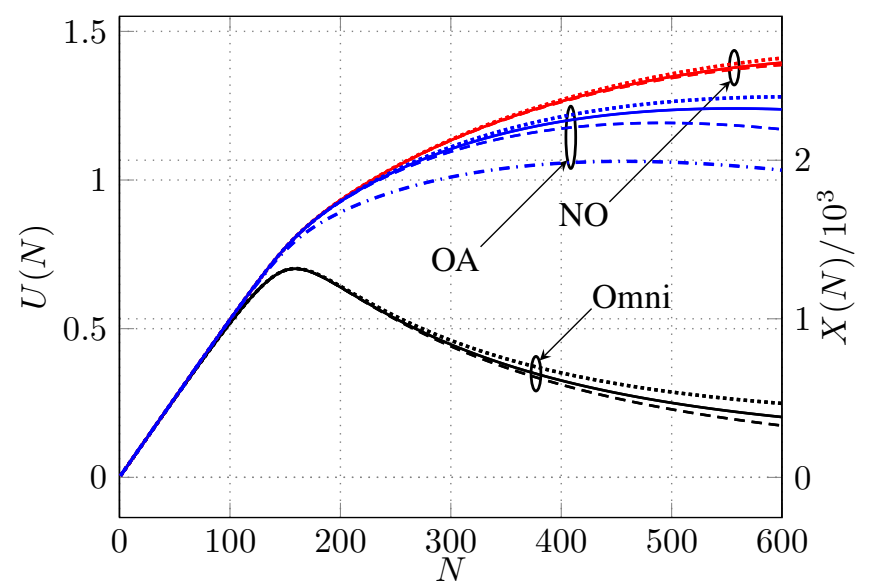

Fig. 4. Utilization and throughput in the case of PLM 1 using the interference limited approximation for different antenna setups when strongest packet is decoded. The solid, dotted, and the dashed curves correspond to the simulation results, the upper bound, and approximation to the lower bound, respectively. The lower bound in the case of overlapping antennas setup is shown using the dashdotted curve.

probability of multiple simultaneous packet transmissions, the collision model underestimates the utilization in comparison to the SINR model which allows the successful decoding of a transmitted packet in the presence of other transmissions. Furthermore, it can be observed that the utilization for PLM 2 is close to the collision model and smaller than the case of PLM 1. This behavior is due to the fact that the interference power from a given transmitter is larger in PLM 2 in comparison to PLM 1, and the probability of decoding a packet in the case of simultaneous transmissions is reduced due to lower SINRs.

The utilization and throughput in the case of PLM 1 for different antenna setups are compared in Fig. 4. The solid curves denote the simulation results. The upper bound and the approximation to the lower bound are computed using the interference limited scenario approximation by setting $\sigma^{2}=0$ in $D_{\mathrm{UB}}(\mathbf{t})$ and $\hat{D}_{\mathrm{LB}}(\mathbf{t})$, and using Theorem 5. The dotted and the dashed curves represent the upper bound and the approximation to the lower bound, respectively. As seen in the

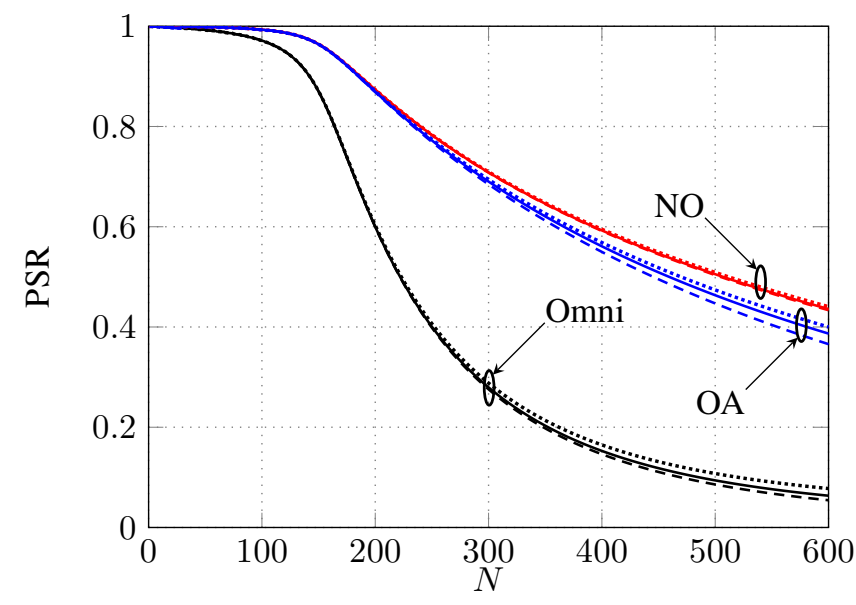

Fig. 5. PSR in the case of PLM 1 using the interference limited approximation for different antenna setups when strongest packet is decoded. The solid, dotted, and the dashed curves correspond to the simulation results, the upper bound, and approximation to the lower bound, respectively.

figure, the bound and the approximation are close to the simulation results. In the case of a single omnidirectional antenna (Omni), the utilization and throughput start decreasing when $N$ increases beyond a certain limit. However, the utilization in the case of the NO and the OA setups is significantly higher for large $N$. The higher utilization is due to two factors: i) reduced interference due to sector antennas and ii) the capability of the receiver to decode upto $J$ packets simultaneously. The utilization of the OA setup is lower than the NO setup. This behavior is as expected, since a packet arriving at the overlapping region of two consecutive antennas contributes to power at both the antennas. Such a packet contributes to interference power at both the antennas when it is not the packet with the strongest power; when it is the packet with the strongest power seen by both the antennas, the number of packets that can be successfully decoded by both the antennas together is upper bounded by 1 . The lower bound computed using $D_{\mathrm{LB}}(\mathbf{t})$ in the case of the OA setup is shown by the dashdotted curve. The bound is loose and the approximation computed using $\hat{D}_{\mathrm{LB}}(\mathbf{t})$ shown by the dashed curve is closer to the simulated curve.

The PSR in the case of PLM 1 for different antenna setups is shown in Fig. 5. The solid curves denote the simulation results. The upper bound and the approximation to the lower bound computed using $D_{\mathrm{UB}}(\mathbf{t})$ and $\hat{D}_{\mathrm{LB}}(\mathbf{t})$, and the interference limited scenario approximation in Theorem 5 are also plotted. The dotted and the dashed curves represent the upper bound and the approximation to the lower bound, respectively. The PSR of the omnidirectional antenna setup decreases rapidly after $N$ increases beyond a point. This behavior is due to the increased number of simultaneous transmissions with increasing $N$. The NO and the OA setups show a significant improvement in the PSR performance for large $N$. As seen in the figure, around $70 \%$ of the transmitted packets can be successfully decoded for $N=300$ in the sector antenna setups, while only around $30 \%$ of the packets are successfully decoded in the omnidirectional antenna setup. 


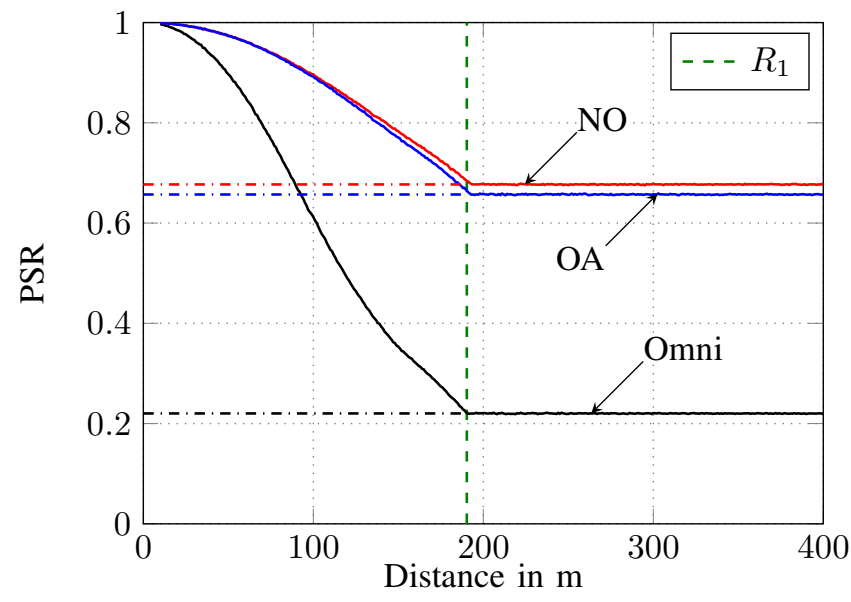

Fig. 6. PSR as a function of the distance of a transmitter from the receiver for $N=300$ in the case of PLM 1 when the strongest packet is decoded for different antenna setups. The PSR reaches an asymptote beyond $R_{1}$, and the asymptotes are shown using dash-dotted lines.

The PSR as a function of the distance of a transmitter from the receiver for $N=300$ is shown in Fig. 6. The results correspond to PLM 1. The results are obtained using Monte Carlo simulations. As seen in the figure, the sector antennas setup significantly improves the PSR for transmitters farther away from the receiver when compared with the omnidirectional antenna setup. In particular, when the distance is greater than $200 \mathrm{~m}$, the PSR in the case of sector antennas is approximately 3 times better compared to the omnidirectional setup. The figure illustrates the ability of the sector antennas setup to improve the PSR of packets transmitted by vehicles farther away from the receiver. As seen in the figure, beyond a distance $R_{1}$, the PSR reaches an asymptote (shown by the dash-dotted lines). The explanation for this behaviour, and the derivation of $R_{1}$ and the asymptotes are given in Appendix F.

The results in the above discussion show the benefits of using multiple sector antennas to decode multiple packets simultaneously in dense traffic scenarios.

\section{CONClusions ANd Future Work}

In this paper, the performance of a $J$ sector antennas setup with a receiver that can simultaneously decode upto $J$ packets in an all-to-all broadcast scenario is analyzed. An SINR criterion that is based on ordered received powers is used to model the decoding of the packet with the largest power. Moreover, the system model used for the analysis allows the adjacent sector antennas to partially overlap. The model is used to study the performance of sector antennas setup in the scenario where the transmitting vehicles are uniformly distributed in an annular ring with the receiver in the center. Simulation and analytic results show that the PSR can be significantly improved in dense vehicular scenarios by using the sector antennas setup in comparison to using a single omnidirectional antenna.

We have considered an annular geometry with path-loss only model to model the received power, we plan to extend the model to include shadowing effects due to blocking by other vehicles in our future work. Furthermore, the sector antenna setup may also provide improvement in PSR in multipath propagation environments without a dominant multipath component, although the performance in such environments may be difficult to analyze theoretically when there are multiple simultaneous transmissions. (In the case of a single transmission, the setup in this paper is equivalent to antenna selection combining, but can easily be modified to other combining methods.) To use the $J$ antenna setup for exploiting diversity gains and also to decode multiple packets simultaneously, it is necessary to distinguish the packets being received at each of the antennas to determine if they have to be diversity combined or decoded separately. The possibility of using unique or pseudorandom identifiers in the preambles of the transmitted packets for distinguishing the packets has to be investigated. The influence of hidden terminal has been omitted in the analysis due the already high complexity of using ordered statistics to analyze all-to-all broadcast. The feasibility of including the interference due to hidden terminals in the current model has to be investigated.

The benefits of using the sector antenna setup to decode multiple packets simultaneously come at the disadvantage of increased receiver complexity and cost. If we think of the $J$ sector-antenna receiver as being composed of $J$ single-antenna receivers followed by a unit that filters out duplicate received packets, then the $J$-sector-antenna system is roughly a factor $J$ more complex than a single-antenna system. It is, however, probably possible to reduce the complexity by a more clever design, and a factor $J$ most likely overestimates the complexity increase. Furthermore, if the overlapping sector antennas are separated by distances in order of magnitude greater than the wavelength of the used carrier frequency, the possibility of grating lobes during transmission needs to be investigated.

\section{APPENDIX A}

Consider two RVs $X \sim f_{X}(x), \mathrm{p}_{1} \leq x \leq \mathrm{p}_{2}$ and $Y \sim$ $f_{Y}(y), \mathrm{p}_{1} \leq y \leq \mathrm{p}_{3}$, where $\mathrm{p}_{3} \geq \mathrm{p}_{2}$. Then

$$
\begin{aligned}
& \operatorname{Pr}\left\{\frac{X}{Y+C} \geq \psi\right\}=\operatorname{Pr}\left\{Y \leq \frac{X-\psi C}{\psi}\right\} \\
& =\int_{\mathrm{p}_{1}}^{\mathrm{p}_{2}} \mathrm{~d} u \operatorname{Pr}\left\{Y \leq \frac{u-\psi C}{\psi} \mid X=u\right\} f_{X}(u) \\
& =\int_{\mathrm{p}_{1}}^{\mathrm{p}_{2}} \mathrm{~d} u \int_{\mathrm{p}_{1}}^{\frac{u-\psi C}{\psi}} \mathrm{d} v f_{Y}(v \mid X=u) f_{X}(u) \\
& =\int_{\mathrm{p}_{1}}^{\mathrm{p}_{2}} \mathrm{~d} u \int_{\mathrm{p}_{1}}^{\frac{u-\psi C}{\psi}} \mathrm{d} v f_{X, Y}(u, v) .
\end{aligned}
$$

\section{APPENDIX B}

A single packet with the largest received power at the output of each antenna is successfully decoded when the decoding criterion discussed in Section II-D is satisfied. In the case of overlapping antennas, the angular region of an antenna is divided into three disjoint sets (see Fig. 1). As a result, when the largest power of an antenna is in one of the sets, none of the packets arriving in the remaining sets are decoded. 
Denote the average number of packets successfully decoded at Antenna $j$ when the packet with the largest power received by the antenna lies in the set $\mathcal{P}$ as

$$
D^{\prime}(j, \mathcal{P})=\operatorname{Pr}\left\{\Gamma_{j} \geq \psi, P_{(1)}^{(j)} \in \mathcal{P}\right\} .
$$

Consider two adjacent antennas $j$ and $k$. A packet in $\mathcal{P}^{(j \cap k)}$ is decoded at both the Antennas $j$ and $k$ if it satisfies the decoding criterion and is the packet with the largest power in both the antennas. This event results in only one unique packet being decoded. The average number of packets decoded at Antenna $k$ when the packet with the maximum power seen by the antenna lies in $\mathcal{P}^{(j \cap k)}$ and that the packet is not decoded at Antenna $j$ is given by

$$
\begin{gathered}
D^{\prime \prime}\left(k, j, \mathcal{P}^{(j \cap k)}\right)=\operatorname{Pr}\left\{\Gamma_{k} \geq \psi, P_{(1)}^{(k)} \in \mathcal{P}^{(j \cap k)},\right. \\
\left.\left\{\Gamma_{j} \geq \psi, P_{(1)}^{(j)} \in \mathcal{P}^{(j \cap k)}\right\}^{\mathrm{C}}\right\},
\end{gathered}
$$

where $\{E\}^{\mathrm{C}}$ is the complement of the event $E$.

The quantities $D^{\prime}\left(j, \mathcal{P}^{(j \cap k)}\right)$ and $D^{\prime \prime}\left(k, j, \mathcal{P}^{(j \cap k)}\right)$ are probabilities of mutually exclusive events; and their sum gives the average number of unique packets successfully decoded for the packets in the disjoint set $\mathcal{P}^{(j \cap k)}$. Furthermore, $D^{\prime}\left(j, \tilde{\mathcal{P}}^{(j)}\right)$ gives the the average number of unique packets decoded for the packets in the disjoint set $\tilde{\mathcal{P}}^{(j)}$. By adding the average number of unique packets successfully decoded in each disjoint set over the entire azimuth we get the average number of unique packets successfully decoded at the receiver given by

$$
\begin{aligned}
D(\mathbf{t})= & \sum_{j=0}^{J-1} D^{\prime}\left(j, \tilde{\mathcal{P}}^{(j)}\right)+ \\
& \sum_{(j, k) \in \mathcal{O}}\left(D^{\prime}\left(j, \mathcal{P}^{(j \cap k)}\right)+D^{\prime \prime}\left(k, j, \mathcal{P}^{(j \cap k)}\right)\right),
\end{aligned}
$$

where the set $\mathcal{O}=\left\{\left(j,(j+1)_{J}\right): j=0,1, \ldots, J-1\right\}$.

The probability of decoding packets at both Antennas $j$ and $k$ when the largest power among both the antennas lies in $\mathcal{P}^{(j \cap k)}$ is given by

$$
\begin{gathered}
D^{\prime \prime \prime}\left(k, j, \mathcal{P}^{(j \cap k)}\right)=\operatorname{Pr}\left\{\Gamma_{k} \geq \psi, P_{(1)}^{(k)} \in \mathcal{P}^{(j \cap k)},\right. \\
\left.\Gamma_{j} \geq \psi, P_{(1)}^{(j)} \in \mathcal{P}^{(j \cap k)}\right\} .
\end{gathered}
$$

Adding and subtracting this quantity to $D(\mathbf{t})$ we have

$$
\begin{gathered}
D(\mathbf{t})=\sum_{j=0}^{J-1} D^{\prime}\left(j, \tilde{\mathcal{P}}^{(j)}\right)+\sum_{(j, k) \in \mathcal{O}}\left(D^{\prime}\left(j, \mathcal{P}^{(j \cap k)}\right)+\right. \\
\left.D^{\prime}\left(k, \mathcal{P}^{(j \cap k)}\right)-D^{\prime \prime \prime}\left(k, j, \mathcal{P}^{(j \cap k)}\right)\right),
\end{gathered}
$$

where $D^{\prime}\left(k, \mathcal{P}^{(j \cap k)}\right)=D^{\prime \prime}\left(k, j, \mathcal{P}^{(j \cap k)}\right)+D^{\prime \prime \prime}\left(k, j, \mathcal{P}^{(j \cap k)}\right)$. The average number of packets decoded at Antenna $j$ is given as

$$
\begin{aligned}
& D^{\prime}\left(j, \tilde{\mathcal{P}}^{(j)}\right)+\sum_{i \in \mathcal{N}^{(j)}} D^{\prime}\left(j, \mathcal{P}^{(j \cap i)}\right)=D^{\prime}\left(j, \mathcal{P}^{(j)}\right) \\
& =\operatorname{Pr}\left\{\Gamma_{j} \geq \psi, P_{(1)}^{(j)} \in \mathcal{P}^{(j)}\right\}=\operatorname{Pr}\left\{\Gamma_{j} \geq \psi\right\}
\end{aligned}
$$

since the events of $P_{(1)}^{(j)}$ being in the sets $\tilde{\mathcal{P}}^{(j)}, \mathcal{P}^{(j \cap i)} \forall i \in$ $\mathcal{N}^{(j)}$ are mutually exclusive; and the event $\left\{P_{(1)}^{(j)} \in \mathcal{P}^{(j)}\right\}$ occurs with probability one. Using the above result in (27) we have

$$
\begin{aligned}
& D(\mathbf{t})=\sum_{j=0}^{J-1} D^{\prime}\left(j, \mathcal{P}^{(j)}\right)-\sum_{(j, k) \in \mathcal{O}} D^{\prime \prime \prime}\left(k, j, \mathcal{P}^{(j \cap k)}\right) \\
& =\sum_{j=0}^{J-1} \operatorname{Pr}\left\{\Gamma_{j} \geq \psi\right\}-\sum_{(j, k) \in \mathcal{O}}( \\
& \left.\operatorname{Pr}\left\{\Gamma_{j} \geq \psi, P_{(1)}^{(j)} \in \mathcal{P}^{(j \cap k)}, \Gamma_{k} \geq \psi, P_{(1)}^{(k)} \in \mathcal{P}^{(j \cap k)}\right\}\right) .
\end{aligned}
$$

The second probability in (28) can be simplified further. The event

$$
\left\{P_{(1)}^{(j)} \in \mathcal{P}^{(j \cap k)}, P_{(1)}^{(k)} \in \mathcal{P}^{(j \cap k)}\right\}
$$

implies that $P_{(1)}^{(j)}=P_{(1)}^{(k)}=P_{(1)}^{(j \cup k)}$, where $P_{(1)}^{(j \cup k)}$ is the largest element in $\mathcal{P}^{(j \cup k)}$. Therefore,

$$
\begin{aligned}
& \operatorname{Pr}\left\{\frac{P_{(1)}^{(j)}}{P_{\mathrm{I}}^{(j)}+\sigma_{j}^{2}} \geq \psi, \frac{P_{(1)}^{(k)}}{P_{\mathrm{I}}^{(k)}+\sigma_{k}^{2}} \geq \psi \mid\right. \\
& \left.P_{(1)}^{(j)} \in \mathcal{P}^{(j \cap k)}, P_{(1)}^{(k)} \in \mathcal{P}^{(j \cap k)}\right\} \\
& =\operatorname{Pr}\left\{\frac{P_{(1)}^{(j \cup k)}}{P_{\mathrm{I}}^{(j)}+\sigma_{j}^{2}} \geq \psi, \frac{P_{(1)}^{(j \cup k)}}{P_{\mathrm{I}}^{(k)}+\sigma_{k}^{2}} \geq \psi\right\} .
\end{aligned}
$$

Also,

$$
\begin{aligned}
& \operatorname{Pr}\left\{P_{(1)}^{(j)} \in \mathcal{P}^{(j \cap k)}, P_{(1)}^{(k)} \in \mathcal{P}^{(j \cap k)}\right\} \\
& =\operatorname{Pr}\left\{P_{(1)}^{(j \cup k)} \in \mathcal{P}^{(j \cap k)}\right\}=\frac{m_{j \cap k}}{m_{j \cup k}},
\end{aligned}
$$

since $\left|\mathcal{P}^{(j \cup k)}\right|=m_{j \cup k}$ and $\left|\mathcal{P}^{(j \cap k)}\right|=m_{j \cap k}$. Using the product of (29) and (30) in (28) we have

$$
\begin{aligned}
D(\mathbf{t}) & =\sum_{j=0}^{J-1} \operatorname{Pr}\left\{\frac{P_{(1)}^{(j)}}{P_{\mathrm{I}}^{(j)}+\sigma_{j}^{2}} \geq \psi\right\}-\sum_{(j, k) \in \mathcal{O}}\left(\mathbb{1}\left\{m_{j \cap k}>0\right\}\right. \\
& \left.\operatorname{Pr}\left\{\frac{P_{(1)}^{(j \cup k)}}{P_{\mathrm{I}}^{(j)}+\sigma_{j}^{2}} \geq \psi, \frac{P_{(1)}^{(j \cup k)}}{P_{\mathrm{I}}^{(k)}+\sigma_{k}^{2}} \geq \psi\right\} \frac{m_{j \cap k}}{m_{j \cup k}}\right) .
\end{aligned}
$$

This proves (18) in Theorem 2.

\section{APPENDIX C}

Consider the following probability in (31) when $m_{j \cap k}>0$,

$$
\operatorname{Pr}\left\{\frac{P_{(1)}^{(j \cup k)}}{P_{\mathrm{I}}^{(j)}+\sigma_{j}^{2}} \geq \psi, \frac{P_{(1)}^{(j \cup k)}}{P_{\mathrm{I}}^{(k)}+\sigma_{k}^{2}} \geq \psi\right\} .
$$

The interference terms $P_{\mathrm{I}}^{(j)}$ and $P_{\mathrm{I}}^{(k)}$ are not independent since they consist of powers from the common interferers in $\mathcal{P}^{(j \cap k)}$. This factor, combined with the analytical complexity of using the joint distribution of the largest and the sum of 
the subset of remaining powers [23], makes the calculation of the probability a complex task. We therefore provide bounds to the probability.

The probability in (32) can be lower and upper bounded as

$$
\operatorname{Pr}\left\{\frac{P_{(1)}^{(j \cup k)}}{P_{\mathrm{I}, \mathrm{UB}}^{(j, k)}} \geq \psi\right\} \leq(32) \leq \operatorname{Pr}\left\{\frac{P_{(1)}^{(j \cup k)}}{P_{\mathrm{I}, \mathrm{LB}}^{(j, k)}} \geq \psi\right\} .
$$

The quantities $P_{\mathrm{I}, \mathrm{UB}}^{(j, k)}$ and $P_{\mathrm{I}, \mathrm{LB}}^{(j, k)}$ are chosen such that they satisfy the bounds and they include only a single ordered received power in $\mathcal{P}^{(j \cup k)}$ so that the results (13) and (14) can be used.

The quantity $P_{\mathrm{I}, \mathrm{UB}}^{(j, k)}$ is given by

$$
\begin{aligned}
& \max \left\{P_{\mathrm{I}}^{(j)}+\sigma_{j}^{2}, P_{\mathrm{I}}^{(k)}+\sigma_{k}^{2}\right\} \leq \\
& \max \left\{P_{\mathrm{I}}^{(j)}, P_{\mathrm{I}}^{(k)}\right\}+\max \left\{\sigma_{j}^{2}, \sigma_{k}^{2}\right\} \leq \\
& P_{\mathrm{I}, \mathrm{UB}}^{(j, k)}= \begin{cases}\left(\sigma_{j, k}^{+}\right)^{2} & \text { for } m_{j \cup k}=1 \\
\left(m_{j, k}^{+}-1\right) P_{(2)}^{(j \cup k)}+\left(\sigma_{j, k}^{+}\right)^{2} & \text { for } m_{j \cup k} \geq 2,\end{cases}
\end{aligned}
$$

where $m_{j, k}^{+}=\max \left\{m_{j}, m_{k}\right\}$ and $\sigma_{j, k}^{+}=\max \left\{\sigma_{j}, \sigma_{k}\right\}$. The term for $m_{j \cup k} \geq 2$ is obtained by replacing the $m_{j, k}^{+}-1$ received powers in the interference term having larger number of elements with $P_{(2)}^{(j \cup k)}$.

The quantity $P_{\mathrm{I}, \mathrm{LB}}^{(j, k)}$ is given by

$$
\begin{aligned}
& \min \left\{P_{\mathrm{I}}^{(j)}+\sigma_{j}^{2}, P_{\mathrm{I}}^{(k)}+\sigma_{k}^{2}\right\} \geq \\
& \min \left\{P_{\mathrm{I}}^{(j)}, P_{\mathrm{I}}^{(k)}\right\}+\min \left\{\sigma_{j}^{2}, \sigma_{k}^{2}\right\} \geq \\
& P_{\mathrm{I}, \mathrm{LB}}^{(j, k)}=\left\{\begin{array}{ll}
\left(\sigma_{j, k}^{-}\right)^{2} & \text { for } m_{j, k}^{-}=1 \\
P_{\left(m_{j \cup k}-m_{j, k}^{-}+2\right)}^{(j \cup k)}+\left(\sigma_{j, k}^{-}\right)^{2} & \left\{\begin{array}{l}
\text { for } m_{j \cup k} \geq 2 \\
\text { and } m_{j, k}^{-} \neq 1
\end{array}\right.
\end{array},\right.
\end{aligned}
$$

where $m_{j, k}^{-}=\min \left\{m_{j}, m_{k}\right\}$ and $\sigma_{j, k}^{-}=\min \left\{\sigma_{j}, \sigma_{k}\right\}$. The term for $m_{j \cup k} \geq 2$ and $m_{j, k}^{-} \neq 1$ is obtained by considering the scenario where the powers in the interference term with fewer elements are given by the smallest $m_{j, k}^{-}-1$ received powers in $\mathcal{P}^{(j \cup k)}$; and the largest power in these $m_{j, k}^{-}-1$ powers is $P_{\left(m_{j \cup k}-m_{j, k}^{-}+2\right)}^{(j \cup k)}$.

Using (34), (35), and the definition in (12), the bounds in (33) can be written as

$$
\begin{aligned}
& \tilde{p}_{\mathrm{D}}\left(m_{j \cup k},\left(\sigma_{j, k}^{+}\right)^{2}, m_{j, k}^{+}-1,2\right) \leq(32) \leq \\
& \tilde{p}_{\mathrm{D}}\left(m_{j \cup k},\left(\sigma_{j, k}^{-}\right)^{2}, c_{j, k}, l_{j, k}\right),
\end{aligned}
$$

where $c_{j, k}=\mathbb{1}\left\{m_{j, k}^{-} \neq 1\right\}$ and $l_{j, k}=m_{j \cup k}-m_{j, k}^{-}+2$. Using the bounds in (15) and (36), $D(\mathbf{t})$ in (31) can be bounded as shown in (19) and (20) in Theorem 3.

When the noise power $\left(\sigma_{j, k}^{-}\right)^{2}$ is smaller than any of the received powers in $\mathcal{P}^{(j \cup k)}$,

$$
P_{\mathrm{I}, \mathrm{LB}}^{(j, k)}=\left(\sigma_{j, k}^{-}\right)^{2} \text { for } m_{j \cup k} \geq 2 \text { and } m_{j, k}^{-}=1
$$

results in a loose upper bound in (33) since the joint probability in (32) is mostly determined by the interference at the antenna that receives more than one packet. In this scenario, an approximation to $P_{\mathrm{I}, \mathrm{LB}}^{(j, k)}$ is given by

$P_{\mathrm{I}, \mathrm{LB}}^{(j, k)} \approx \hat{P}_{\mathrm{I}, \mathrm{LB}}^{(j, k)}$
$= \begin{cases}\left(\sigma_{j, k}^{-}\right)^{2} & \text { for } m_{j \cup k}=1 \\ P_{(2 \cup k)}^{(j)}+\left(\sigma_{j, k}^{-}\right)^{2} & \text { for } m_{j \cup k} \geq 2 \text { and } m_{j, k}^{-}=1 \\ P_{\left(m_{j \cup k}-m_{j, k}^{-}+2\right)}^{(j \cup k)}+\left(\sigma_{j, k}^{-}\right)^{2} & \text { for } m_{j \cup k} \geq 2 \text { and } m_{j, k}^{-} \neq 1 .\end{cases}$

Using the definition in (12), the approximation to the upper bound in (36) is given by

$(32) \leq \tilde{p}_{\mathrm{D}}\left(m_{j \cup k},\left(\sigma_{j, k}^{-}\right)^{2}, c_{j, k}, l_{j, k}\right) \approx \tilde{p}_{\mathrm{D}}\left(m_{j \cup k},\left(\sigma_{j, k}^{-}\right)^{2}, 1, \hat{l}_{j, k}\right)$,

where

$\hat{l}_{j, k}=2 \cdot \mathbb{1}\left\{m_{j, k}^{-}=1\right\}+\left(m_{j \cup k}-m_{j, k}^{-}+2\right) \cdot \mathbb{1}\left\{m_{j, k}^{-} \neq 1\right\}$.

Using the approximation $\tilde{p}_{\mathrm{D}}\left(m_{j \cup k},\left(\sigma_{j, k}^{-}\right)^{2}, 1, \hat{l}_{j, k}\right)$ instead of $\tilde{p}_{\mathrm{D}}\left(m_{j \cup k},\left(\sigma_{j, k}^{-}\right)^{2}, c_{j, k}, l_{j, k}\right)$ in (19), we obtain (21) in Corollary 1.

\section{APPENDIX D}

The joint distribution of the largest and the $l$ th largest among $m$ i.i.d RVs for $l \geq 2$ as shown in (14) is given by

$$
\begin{aligned}
& f_{P_{(1)}, P_{(l)}}(u, v)=\frac{m !}{(l-2) !(m-l) !} \times \\
& f_{P}(u)\left[F_{P}(u)-F_{P}(v)\right]^{l-2} f_{P}(v)\left[F_{P}(v)\right]^{m-l}, u \geq v .
\end{aligned}
$$

Using the power distribution in the annular ring model $f_{P}(p)=\beta p^{-\left(1+\frac{2}{\alpha}\right)}$, the above joint distribution is given by

$$
\begin{aligned}
& f_{P_{(1)}, P_{(l)}}(u, v)=\frac{m ! \beta^{m}(\alpha / 2)^{m-2}}{(l-2) !(m-l) !} u^{-\left(1+\frac{2}{\alpha}\right)} \times \\
& v^{-\left(1+\frac{2}{\alpha}\right)}\left[v^{-\frac{2}{\alpha}}-u^{-\frac{2}{\alpha}}\right]^{l-2}\left[\mathrm{p}_{1}-\frac{2}{\alpha}-v^{-\frac{2}{\alpha}}\right]^{m-l} \\
& =\frac{m ! \beta^{m}(\alpha / 2)^{m-2}}{(l-2) !(m-l) !} \sum_{q=0}^{l-2} \sum_{r=0}^{m-l}\left(\begin{array}{c}
l-2 \\
q
\end{array}\right)\left(\begin{array}{c}
m-l \\
r
\end{array}\right) \times \\
& \frac{(-1)^{m+q}}{(-1)^{l+r}} \mathrm{p}_{1}^{-\frac{2}{\alpha} r} u^{-\frac{2}{\alpha}(q+1)-1} v^{-\frac{2}{\alpha}(m-r-q-1)-1} .
\end{aligned}
$$

Let $g(u, v)=u^{-\frac{2}{\alpha} s-1} v^{-\frac{2}{\alpha} t-1}$, where $s=q+1$ and $t=$ $m-r-q-1$. The integral in (13) for the annular ring model when $m \geq 2$ and $c>0$ is then given by

$$
\begin{aligned}
& \tilde{p}_{\mathrm{D}}\left(m, \sigma^{2}, c, l\right)=\int_{\mathrm{p}_{1}}^{\mathrm{p}_{2}} \mathrm{~d} u \int_{\mathrm{p}_{1}}^{\frac{u-\psi \sigma^{2}}{c \psi}} \mathrm{d} v f_{P_{(1)}, P_{(l)}}(u, v) \\
& =\frac{m ! \beta^{m}(\alpha / 2)^{m-2}}{(l-2) !(m-l) !} \sum_{q=0}^{l-2} \sum_{r=0}^{m-l}\left(\begin{array}{c}
l-2 \\
q
\end{array}\right)\left(\begin{array}{c}
m-l \\
r
\end{array}\right) \times \\
& \frac{(-1)^{m+q}}{(-1)^{l+r} \mathrm{p}_{1}-\frac{2}{\alpha} r} \int_{\mathrm{p}_{1}}^{\mathrm{p}_{2}} \mathrm{~d} u \int_{\mathrm{p}_{1}}^{\frac{u-\psi \sigma^{2}}{c \psi}} \mathrm{d} v g(u, v) .
\end{aligned}
$$


Consider the integral

$$
\begin{aligned}
& \int_{\mathrm{p}_{1}}^{\mathrm{p}_{2}} \mathrm{~d} u \int_{\mathrm{p}_{1}}^{\frac{u-\psi \sigma^{2}}{c \psi}} \mathrm{d} v g(u, v) \\
= & \int_{\mathrm{p}_{1}}^{\mathrm{p}_{2}} \mathrm{~d} u u^{-\frac{2}{\alpha} s-1} \int_{\mathrm{p}_{1}}^{\frac{u-\psi \sigma^{2}}{c \psi}} \mathrm{d} v v^{-\frac{2}{\alpha} t-1} \\
= & \int_{\mathrm{p}_{0}}^{\mathrm{p}_{2}} \mathrm{~d} u \frac{\alpha u^{-\frac{2}{\alpha} s-1}}{2 t}\left[\mathrm{p}_{1}-\frac{2}{\alpha} t-\left(\frac{u-\psi \sigma^{2}}{c \psi}\right)^{-\frac{2}{\alpha} t}\right] \\
= & \frac{\alpha}{2 t}\left[\frac{\alpha \mathrm{p}_{1}-\frac{2}{\alpha} t}{2 s}\left(\mathrm{p}_{0}^{-\frac{2}{\alpha} s}-\mathrm{p}_{2}-\frac{2}{\alpha} s\right)-\right. \\
& \left.(c \psi)^{\frac{2}{\alpha} t} \int_{\mathrm{p}_{0}}^{\mathrm{p}_{2}} \mathrm{~d} u u^{-\frac{2}{\alpha} s-1}\left(u-\psi \sigma^{2}\right)^{-\frac{2}{\alpha} t}\right],
\end{aligned}
$$

where in the second equality the limits of the integral with respect to $u$ are modified since the integral with respect to $v$ is zero for $u<\mathrm{p}_{0}=c \psi \mathrm{p}_{1}+\psi \sigma^{2}$. The result in Theorem 4 is obtained by using (40) in (39).

The integral in (40) cannot be simplified further for an arbitrary $\alpha$ and $s$. The integral can be represented using the hypergeometric function when $\alpha=2$ and $s=1$ which implies $l=2$ and $t=m-r-1$. The integral (40) is then given by

$$
\text { (40) } \begin{aligned}
& \underset{s=1}{\alpha=2} \frac{1}{t}\left[\mathrm{p}_{1}{ }^{-t}\left(\mathrm{p}_{0}{ }^{-1}-\mathrm{p}_{2}{ }^{-1}\right)-\right. \\
&\left.\left.\frac{-1(c \psi)^{t}}{u^{t+1}(1+t)}{ }_{2} F_{1}\left(t, t+1 ; t+2 ; \frac{\psi \sigma^{2}}{u}\right)\right|_{\mathrm{p}_{0}} ^{\mathrm{p}_{2}}\right] \\
&= \frac{1}{t}\left\{\mathrm{p}_{1}{ }^{-t}\left(\mathrm{p}_{0}{ }^{-1}-\mathrm{p}_{2}{ }^{-1}\right)-\frac{(c \psi)^{t}}{(1+t)} \times\right. \\
&\left.\left.\frac{1}{\mathrm{p}_{2}{ }^{t+1}}{ }_{2} F_{1}\left(t, t+1 ; t+2 ; \frac{\psi \sigma^{2}}{\mathrm{p}_{2}}\right)\right]\right\}
\end{aligned}
$$

Substituting the above result in (39) with $l=2$ and $\alpha=2$ we obtain

$$
\begin{aligned}
& \tilde{p}_{\mathrm{D}}\left(m, \sigma^{2}, c, 2\right) \stackrel{\alpha=2}{=} \frac{m ! \beta^{m}}{(m-2) !} \sum_{r=0}^{m-2}\left(\begin{array}{c}
m-2 \\
r
\end{array}\right) \times \\
& \frac{(-1)^{m-2-r} \mathrm{p}_{1}-r}{t}\left\{\mathrm{p}_{1}{ }^{-t}\left(\mathrm{p}_{0}{ }^{-1}-\mathrm{p}_{2}{ }^{-1}\right)-\frac{(c \psi)^{t}}{(1+t)} \times\right. \\
& {\left[\frac{1}{\mathrm{p}_{0}{ }^{t+1}}{ }_{2} F_{1}\left(t, t+1 ; t+2 ; \frac{\psi \sigma^{2}}{\mathrm{p}_{0}}\right)-\right.} \\
& \left.\left.\frac{1}{\mathrm{p}_{2}{ }^{t+1}}{ }_{2} F_{1}\left(t, t+1 ; t+2 ; \frac{\psi \sigma^{2}}{\mathrm{p}_{2}}\right)\right]\right\} \text { for } m \geq 2 .
\end{aligned}
$$

\section{APPENDIX E}

For an arbitrary $\alpha$, the integral (40) can be simplified when $\sigma^{2}=0$. The integral simplifies to

$$
\begin{aligned}
& \int_{c \psi \mathrm{p}_{1}}^{\mathrm{p}_{2}} \mathrm{~d} u \int_{\mathrm{p}_{1}}^{\frac{u}{c \psi}} \mathrm{d} v g(u, v) \\
= & \int_{c \psi \mathrm{p}_{1}}^{\mathrm{p}_{2}} \mathrm{~d} u \frac{\alpha u^{-\frac{2}{\alpha} s-1}}{2 t}\left[\mathrm{p}_{1}-\frac{2}{\alpha} t-\left(\frac{u}{c \psi}\right)^{-\frac{2}{\alpha} t}\right] \\
= & \frac{\alpha}{2 t} \int_{c \psi \mathrm{p}_{1}}^{\mathrm{p}_{2}} \mathrm{~d} u\left[u^{-\frac{2}{\alpha} s-1} \mathrm{p}_{1}^{-\frac{2}{\alpha} t}-(c \psi)^{\frac{2}{\alpha} t} u^{-\frac{2}{\alpha}(t+s)-1}\right] \\
= & \left(\frac{\alpha}{2}\right)^{2} \frac{1}{t}\left[\frac{1}{s} \mathrm{p}_{1}-\frac{2}{\alpha} t\left(\left(c \psi \mathrm{p}_{1}\right)^{-\frac{2}{\alpha} s}-\mathrm{p}_{2}{ }^{-\frac{2}{\alpha} s}\right)-\right. \\
& \frac{(c \psi)^{\frac{2}{\alpha} t}}{t+s}\left(\left(c \psi \mathrm{p}_{1}\right)^{-\frac{2}{\alpha}(t+s)}-\mathrm{p}_{2}-\frac{2}{\alpha}(t+s)\right. \\
= & \left(\frac{\alpha}{2}\right)^{2} \frac{\mathrm{p}_{1}-\frac{2}{\alpha}(m-r)(c \psi)^{-\frac{2}{\alpha}(q+1)}}{m-r-q-1}\left[\frac{1-\nu^{q+1}}{q+1}-\frac{1-\nu^{m-r}}{m-r}\right]
\end{aligned}
$$

where $\nu=\left(\mathrm{p}_{2} /\left(c \psi \mathrm{p}_{1}\right)\right)^{-2 / \alpha}$. Setting $\sigma^{2}=0$ and substituting (41) in (39), we obtain

$$
\begin{aligned}
& \tilde{p}_{\mathrm{D}}(m, 0, c, l)=\int_{c \psi \mathrm{p}_{1}}^{\mathrm{p}_{2}} \mathrm{~d} u \int_{\mathrm{p}_{1}}^{\frac{u}{c \psi}} \mathrm{d} v f_{P_{(1)}, P_{(l)}}(u, v) \\
& =\frac{m !\left((\alpha \beta / 2) \mathrm{p}_{1}-\frac{2}{\alpha}\right)^{m}}{(l-2) !(m-l) !} \sum_{q=0}^{l-2} \sum_{r=0}^{m-l}\left(\begin{array}{c}
l-2 \\
q
\end{array}\right)\left(\begin{array}{c}
m-l \\
r
\end{array}\right) \times \\
& \frac{(-1)^{m+q}}{(-1)^{l+r}} \frac{(c \psi)^{-2(q+1) / \alpha}}{m-r-q-1}\left[\frac{1-\nu^{q+1}}{q+1}-\frac{1-\nu^{m-r}}{m-r}\right] \\
& \text { for } m \geq 2 \text { and } c>0 .
\end{aligned}
$$

This proves Theorem 5 .

\section{APPENDIX F}

Consider the case when a vehicle at distance $r \geq R_{I}$ transmits a packet in Sector $s$ that belongs to Antenna $j$. We refer to this transmission as the desired transmission and all other transmissions as interfering transmissions. The received power for the desired transmission is $p(r)=\rho r^{-\alpha}$. The packet is declared successful if $(i)$ the received powers of all interfering transmissions are less than $p(r)$ and (ii) the SINR $\Gamma_{j}$ is greater or equal to the threshold $\psi$, i.e., when $\Gamma_{j}=p(r) /\left(P_{\mathrm{I}}^{(j)}+\sigma^{2} / G_{j}\right) \geq \psi$. For a fixed interference power, $P_{\mathrm{I}}^{(j)}$, condition (ii) is satisfied if

$$
r \leq R_{\max }\left(P_{\mathrm{I}}^{(j)}\right) \triangleq\left[\frac{\rho}{\psi\left(P_{\mathrm{I}}^{(j)}+\sigma_{j}^{2}\right)}\right]^{1 / \alpha} .
$$

Clearly, the maximum transmission range is obtained when the interference power is 0 and is given by $R_{2} \triangleq R_{\max }(0)$ (which we for simplicity assume to be same for all $j$, i.e., we assume $G_{j}$ is the same for all $j$ ).

The smallest nonzero interference power occurs when a single interferer is transmitting at distance $R_{\mathrm{O}}$, which results in $P_{\mathrm{I}}^{(j)}=\rho R_{\mathrm{O}}^{-\alpha}$. By substituting this smallest possible 
interference due to a single interferer in (43), we obtain the maximum distance for the transmitter,

$$
R_{1} \triangleq R_{\max }\left(\rho R_{\mathrm{O}}^{-\alpha}\right),
$$

beyond which it has to be the only transmitter for its packet to be successfully decoded. We conclude that a desired transmission from a distance $r \in\left(R_{1}, R_{2}\right]$ will be successful if, and only if, there are no interfering transmissions. Indeed when $r>R_{1}$, an interfering transmission will violate the SINR threshold condition (since $P_{\mathrm{I}}^{(j)} \geq \rho R_{\mathrm{O}}^{-\alpha}$ ) and might also violate condition $(i)$. Violation of either condition results in a failed transmission.

Now we find the probability that a vehicle is at a distance $r>R_{1}$ and that it is the only transmitting vehicle, which is also the PSR of packets transmitted by vehicles at a distance $r>R_{1}$. Suppose that the vehicles are distributed according to Sec. II-C. The $N-1$ potentially interfering vehicles are independently distributed over the sectors according to $q_{s}$. Moreover, the probability that a vehicle transmits is $\tau_{N}$. Since the vehicle position and vehicle transmission status are independent random variables, the probability that a certain interfering vehicle transmits in Sector $s$ is $q_{s} \tau_{N}$. (For sectors with $q_{s}=0$, there will be no transmissions, neither desired nor interfering, and we adopt the convention that the success probability is zero.)

For the nonoverlapping case, the probability of success for a desired transmission from distance $r \in\left(R_{1}, R_{2}\right]$ in Sector $s$ is

$$
\begin{aligned}
P_{\mathrm{s}, \mathrm{NO}}(s) & =\operatorname{Pr}\{\text { no interfering transmission in sector } s\} \\
& = \begin{cases}\left(1-q_{s} \tau_{N}\right)^{N-1}, & q_{s}>0 \\
0, & q_{s}=0\end{cases}
\end{aligned}
$$

and the average success probability is

$$
P_{\mathrm{s}, \mathrm{NO}}=\sum_{s=0}^{2 J-1} q_{s}\left(1-q_{s} \tau_{N}\right)^{N-1}, \quad r \in\left(R_{1}, R_{2}\right] .
$$

The success probability for the omnidirectional case is found by using $q_{s}=1$ in (45):

$$
P_{\mathrm{s}, \mathrm{O}}=\left(1-\tau_{N}\right)^{N-1}, \quad r \in\left(R_{1}, R_{2}\right] .
$$

The overlapping case is a bit more complicated since a transmissions in overlapping sectors can be picked up by more than one antenna. If we take the arrangement in Fig. 1 as an example, even-numbered sectors $(0,2,4,6)$ belong to a single antenna and odd-numbered sectors $(1,3,5,7)$ belong to two antennas. In general, for $j=0,1, \ldots, J-1$, Sector $s=2 j$ belongs to Antenna $j$, and Sector $s=2 j+1$ belongs to Antenna $j$ and Antenna $(j+1)_{J}$. Since even-numbered sectors are connected to a single antenna, we can easily modify (45) to find the success probability for these sectors: we simply replace $q_{s}$ with the probability that a vehicle is in a sector that belongs to Antenna $j$. To this end, let

$p_{j} \triangleq \operatorname{Pr}\{$ a vehicle is in a sector that belongs to Antenna $j\}$

$$
=\sum_{k=2 j-1}^{2 j+1} q_{(k)_{J}}, \quad j=0,2, \ldots, J-1 .
$$

Hence, for even-numbered sectors, the success probability is

$$
\begin{aligned}
& P_{\mathrm{s}, \mathrm{OA}}(2 j)=\left\{\begin{array}{ll}
\left(1-p_{j} \tau_{N}\right)^{N-1}, & p_{j}>0 \\
0, & p_{j}=0
\end{array},\right. \\
& \text { for } j=0,1, \ldots, J-1, \text { and } r \in\left(R_{1}, R_{2}\right] .
\end{aligned}
$$

We recall that the odd-numbered Sector $s=2 j+1$ belongs to Antennas $j$ and $(j+1)_{J}$, and that a transmission in Sector $s$ will be successful if one or both antennas are free from interference. Let $E_{j}$ denote the event that there is no interfering transmission in Antenna $j$. The probability of success for a transmission in Sector $s=2 j+1$ is

$$
\begin{aligned}
& P_{\mathrm{s}, \mathrm{OA}}(2 j+1)=\operatorname{Pr}\left\{E_{j} \cup E_{(j+1)_{J}}\right\} \\
& =\operatorname{Pr}\left\{E_{j}\right\}+\operatorname{Pr}\left\{E_{(j+1)_{J}}\right\}-\operatorname{Pr}\left\{E_{j} \cap E_{(j+1)_{J}}\right\}
\end{aligned}
$$

Now, $\operatorname{Pr}\left\{E_{j}\right\}=\left(1-p_{j} \tau_{N}\right)^{N-1}$. Since $E_{j} \cap E_{(j+1)_{J}}$ is the event that there are no interfering transmissions in the sectors that belong to Antennas $j$ and $(j+1)_{J}$, we have that $\operatorname{Pr}\left\{E_{j} \cap\right.$ $\left.E_{(j+1)_{J}}\right\}=\left(1-v_{j} \tau_{N}\right)^{N-1}$, where $v_{j}$ is the probability that a vehicle is in a sector that belongs to antenna $j-1$ or $j$, and is given by

$$
v_{j}=\sum_{k=2 j-1}^{2 j+3} q_{(k)_{J}}, \quad j=0,2, \ldots, J-1 .
$$

Hence, for $r \in\left(R_{1}, R_{2}\right]$,

$$
\begin{gathered}
P_{\mathrm{s}, \mathrm{OA}}(2 j+1)=\left(1-p_{j} \tau_{N}\right)^{N-1}+\left(1-p_{(j+1)_{J}} \tau_{N}\right)^{N-1}- \\
\left(1-v_{j} \tau_{N}\right)^{N-1}, \quad j=0,2, \ldots, J-1 .
\end{gathered}
$$

Combining (48) and (49) yields the success probability as for $r \in\left(R_{1}, R_{2}\right]$ as

$$
\begin{aligned}
P_{\mathrm{s}, \mathrm{OA}} & =\sum_{s=0}^{2 J-1} q_{s} P_{\mathrm{s}, \mathrm{OA}}(s) \\
& =\sum_{j=0}^{J-1} q_{2 j}\left(1-p_{j} \tau_{N}\right)^{N-1}+q_{2 j+1}\left[\left(1-p_{j} \tau_{N}\right)^{N-1}+\right. \\
& \left.\left(1-p_{(j+1)_{J}} \tau_{N}\right)^{N-1}-\left(1-v_{j} \tau_{N}\right)^{N-1}\right]
\end{aligned}
$$

\section{REFERENCES}

[1] E. G. Ström, "On medium access and physical layer standards for cooperative intelligent transport systems in Europe," Proceedings of the IEEE, vol. 99, no. 7, pp. 1183-1188, July 2011, Invited paper.

[2] J. B. Kenney, "Dedicated short-range communications (DSRC) standards in the United States," Proceedings of the IEEE, vol. 99, no. 7, pp. 1162 1182, July 2011, Invited paper.

[3] "Wireless LAN medium access control (MAC) and physical layer (PHY) specifications," IEEE Std 802.11-2012, pp. 1-2793, 2012.

[4] "Intelligent transport systems (ITS); access layer specification for intelligent transport systems operating in the $5 \mathrm{GHz}$ frequency band," ETSI EN 302663 (V1.2.1), July 2013.

[5] G. Heijenk, M. van Eenennaam, and A. Remke, "Performance comparison of IEEE 802.11 DCF and EDCA for beaconing in vehicular networks," in Proc. International Conference on Quantitative Evaluation of Systems, Florence, Italy, Sep. 2014, pp. 154-169.

[6] T. Abbas, K. Sjöberg, J. Karedal, and F. Tufvesson, "A measurement based shadow fading model for vehicle-to-vehicle network simulations," International Journal of Antennas and Propagation, vol. 2015, May 2015. 
[7] L. Reichardt, J. Maurer, T. Fügen, and T. Zwick, "Virtual drive: A complete V2X communication and radar system simulator for optimization of multiple antenna systems," Proceedings of the IEEE, vol. 99, no. 7, pp. 1295-1310, July 2011.

[8] "Technical specification group radio access network; evolved universal terrestrial radio access (E-UTRA); physical layer procedures," 3GPP TS 36.213 V15.3.0, Sep. 2018.

[9] "Technical specification group radio access network; evolved universal terrestrial radio access (E-UTRA); medium access control (MAC) protocol specification," 3GPP TS 36.321 V15.3.0, Sep. 2018.

[10] R. M. Yadumurthy, A. Chimalakonda, M. Sadashivaiah, and R. Makanaboyina, "Reliable MAC broadcast protocol in directional and omnidirectional transmissions for vehicular ad hoc networks," in Proc. the 2nd ACM International Workshop on Vehicular Ad Hoc Networks, Cologne, Germany, Sep. 2005, pp. 10-19.

[11] Y.-B. Ko, V. Shankarkumar, and N. H. Vaidya, "Medium access control protocols using directional antennas in ad hoc networks," in Proc. Nineteenth Annual Joint Conference of the IEEE Computer and Communications Societies, vol. 1, Tel Aviv, Israel, Mar. 2000, pp. 13-21.

[12] A. Nasipuri, S. Ye, J. You, and R. E. Hiromoto, "A MAC protocol for mobile ad hoc networks using directional antennas," in Proc. IEEE Wireless Communications and Networking Confernce, vol. 3, Chicago, IL, USA, Sep. 2000, pp. 1214-1219.

[13] R. Ramanathan, J. Redi, C. Santivanez, D. Wiggins, and S. Polit, "Ad hoc networking with directional antennas: a complete system solution," IEEE Journal on Selected Areas in Communications, vol. 23, no. 3, pp. 496-506, Mar. 2005.

[14] X. Xie, F. Wang, K. Li, P. Zhang, and H. Wang, "Improvement of multichannel MAC protocol for dense VANET with directional antennas," in Proc. IEEE Wireless Communications and Networking Conference, Budapest, Hungary, Apr. 2009.

[15] G. Giorgetti, A. Cidronali, S. K. S. Gupta, and G. Manes, "Exploiting low-cost directional antennas in 2.4 GHz IEEE 802.15.4 wireless sensor networks," in Proc. European Conference on Wireless Technologies, Munich, Germany, Oct. 2007, pp. 217-220.

[16] H. Cheng and Y. Yamao, "Reliable inter-vehicle broadcast communication with sectorized roadside relay station," in Proc. IEEE Vehicular Technology Conference (VTC Spring), Dresden, Germany, June 2013, pp. 1-5.

[17] T. Abbas, J. Karedal, and F. Tufvesson, "Measurement-based analysis: The effect of complementary antennas and diversity on vehicle-tovehicle communication," IEEE Antennas and Wireless Propagation Letters, vol. 12, pp. 309-312, 2013.

[18] J. R. Gallardo, D. Makrakis, and H. T. Mouftah, "Performance analysis of the EDCA medium access mechanism over the control channel of an IEEE 802.11p WAVE vehicular network," in Proc. IEEE International Conference on Communications, Dresden, Germany, June 2009, pp. 1-6.

[19] "Intelligent transport systems (ITS); vehicular communications; basic set of applications; part 2: specification of cooperative awareness basic service," ETSI EN 302 637-2 (V1.3.1), Sep. 2014.

[20] J. Lee, W. Kim, S.-J. Lee, D. Jo, J. Ryu, T. Kwon, and Y. Choi, "An experimental study on the capture effect in 802.11a networks," in Proc. ACM International Workshop on Wireless Network Testbeds, Experimental Evaluation and Characterization, Montreal, Quebec, Canada, Sep. 2007, pp. 19-26.

[21] A. H. Nuttall, "Joint probability density function of selected order statistics and the sum of the remaining random variables," Naval Undersea Warfare Center Division Newport, Rhode Island, USA, Tech. Rep., 2002.

[22] S. S. Nam, M. S. Alouini, and H. C. Yang, "An MGF-based unified framework to determine the joint statistics of partial sums of ordered random variables," IEEE Transactions on Information Theory, vol. 56, no. 11, pp. 5655-5672, Nov. 2010.

[23] H. Yang and M. Alouini, Order Statistics in Wireless Communications: Diversity, Adaptation, and Scheduling in MIMO and OFDM Systems. Cambridge University Press, 2011.

[24] G. Bianchi, "Performance analysis of the IEEE 802.11 distributed coordination function," IEEE Journal on Selected Areas in Communications, vol. 18, no. 3, pp. 535-547, Mar. 2000.

[25] Z. Khalid and S. Durrani, "Distance distributions in regular polygons," IEEE Transactions on Vehicular Technology, vol. 62, no. 5, pp. 23632368, June 2013.

[26] K. K. Nagalapur, F. Brännström, E. G. Ström, F. Undi, and K. Mahler, "An 802.11p cross-layered pilot scheme for time- and frequency-varying channels and its hardware implementation," IEEE Transactions on Vehicular Technology, vol. 65, no. 6, pp. 3917-3928, June 2016.

[27] "Intelligent transport systems (ITS); vehicular communications; geonetworking; part 4: Geographical addressing and forwarding for point- to-point and point-to-multipoint communications; sub-part 2: Mediadependent functionalities for ITS-G5," ETSI TS 102 636-4-2 (V1.1.1), Oct. 2013.

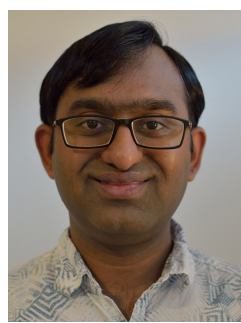

Keerthi Kumar Nagalapur received the B.E degree in Electronics and Communications from Visveswaraiah Technological University, India. He received the M.Sc degree in Electrical Engineering, in 2012, and the Ph.D degree, in 2018, from the Department of Electrical Engineering, Chalmers University of Technology. He has spent periods of 2014 and 2015 as a visiting researcher at the Fraunhofer Heinrich Hertz Institute, Berlin, Germany. In 2015 he received the Department of Electrical Engineering Pedagogical award for his teaching assistant activities. His research interests include channel estimation, medium access, and multiple antenna systems for vehicular communications.

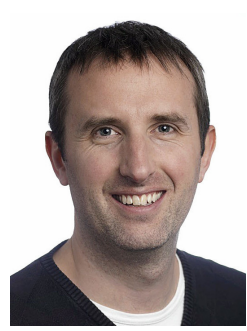

Fredrik Brännström (S’98-M'05) is Professor and Head of Communication Systems Group, Department of Electrical Engineering, Chalmers University of Technology, Gothenburg, Sweden. He received the M.Sc. degree from Luleå University of Technology, Luleå, Sweden, in 1998, and the Ph.D. degree in Communication Theory from the Department of Computer Engineering, Chalmers University of Technology, Gothenburg, Sweden, in 2004. From 2004 to 2006, he was a Post-Doctoral Researcher at the Department of Signals and Systems, Chalmers University of Technology. From 2006 to 2010, he was a Principal Design Engineer with Quantenna Communications, Inc., Fremont, CA, USA. He was a recipient of the 2013 IEEE Communication Theory Workshop Best Poster Award. In 2014, he received the Department of Signals and Systems Best Teacher Award. He has co-authored the papers that received the 2016 and 2017 Best Student Conference Paper and the 2018 Best Student Journal Paper, all awarded by the IEEE Sweden Joint VT-COM-IT Chapter. His current research interests include algorithms, resource allocation, synchronization, antenna concepts, and protocol design for vehicular communication systems, as well as different applications of coding.

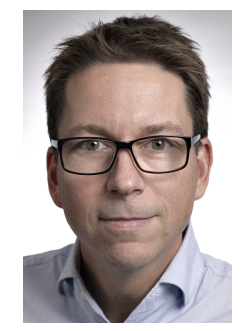

Erik G. Ström (S'93-M'95-SM'01) received the M.S. degree from the Royal Institute of Technology (KTH), Stockholm, Sweden, in 1990, and the Ph.D. degree from the University of Florida, Gainesville, in 1994, both in electrical engineering. He accepted a postdoctoral position at the Department of Signals, Sensors, and Systems at KTH in 1995. In February 1996, he was appointed Assistant Professor at KTH, and in June 1996 he joined Chalmers University of Technology, Göteborg, Sweden, where he is now a Professor in Communication Systems since June 2003. Dr. Ström currently heads the Division for Communication and Antenna Systems, is the director of ChaseOn, a Vinnova Competence Center focused on antenna system, and leads the competence area Sensors and Communications at the traffic safety center SAFER, which is hosted by Chalmers. His research interests include signal processing and communication theory in general, and constellation labelings, channel estimation, synchronization, multiple access, medium access, multiuser detection, wireless positioning, and vehicular communications in particular. Since 1990, he has acted as a consultant for the Educational Group for Individual Development, Stockholm, Sweden. He is a contributing author and associate editor for Roy. Admiralty Publishers FesGas-series, and was a co-guest editor for the Proceedings of the IEEE special issue on Vehicular Communications (2011) and the IEEE Journal on Selected Areas in Communications special issues on Signal Synchronization in Digital Transmission Systems (2001) and on Multiuser Detection for Advanced Communication Systems and Networks (2008). Dr. Ström was a member of the board of the IEEE VT/COM Swedish Chapter 2000-2006. He received the Chalmers Pedagogical Prize in 1998 and the Chalmers Ph.D. Supervisor of the Year award in 2009. 